ESAIM: M2AN 55 (2021) 969-1003

https://doi.org/10.1051/m2an/2021002
ESAIM: Mathematical Modelling and Numerical Analysis

www.esaim-m2an.org

\title{
FINITE VOLUME APPROXIMATION OF A TWO-PHASE TWO FLUXES DEGENERATE CAHN-HILLIARD MODEL
}

\author{
Clément Cancès ${ }^{1, *}$ And Flore NaBeT ${ }^{2}$
}

\begin{abstract}
We study a time implicit Finite Volume scheme for degenerate Cahn-Hilliard model proposed in [W. E and P. Palffy-Muhoray, Phys. Rev. E 55 (1997) R3844-R3846] and studied mathematically by the authors in [C. Cancès, D. Matthes and F. Nabet, Arch. Ration. Mech. Anal. 233 (2019) 837-866]. The scheme is shown to preserve the key properties of the continuous model, namely mass conservation, positivity of the concentrations, the decay of the energy and the control of the entropy dissipation rate. This allows to establish the existence of a solution to the nonlinear algebraic system corresponding to the scheme. Further, we show thanks to compactness arguments that the approximate solution converges towards a weak solution of the continuous problems as the discretization parameters tend to 0 . Numerical results illustrate the behavior of the numerical model.
\end{abstract}

Mathematics Subject Classification. 65M12, 65M08, 76T99, 35K52, 35K65.

Received May 4, 2020. Accepted January 15, 2021.

\section{The Two-Phase two FluXes Degenerate Cahn-Hilliard Model}

The goal of this paper is to propose a convergent finite volume discretization for a degenerate Cahn-Hilliard model proposed by E and Palffy-Muhoray [32] and studied in [8] by the authors. Before considering the numerical scheme, let us describe and discuss the continuous model.

\subsection{The continuous model}

We consider a mixture made of two incompressible phases evolving in a bounded and connected polygonal open subset $\Omega$ of $\mathbb{R}^{2}$ and on a time interval $[0, T]$, where $T$ is an arbitrary finite time horizon. The composition of the fluid is described by the volume fractions $\boldsymbol{c}=\left(c_{1}, c_{2}\right)$ of the two phases. Since the whole volume $\Omega$ is occupied by the two phases, the following constraint on the $c_{i}$ holds

$$
c_{1}+c_{2}=1 \quad \text { in }(0, T) \times \Omega .
$$

The evolution of the volume fractions is prescribed by the following partial differential equations

$$
\partial_{t} c_{i}-\nabla \cdot\left(\frac{c_{i}}{\eta_{i}} \nabla\left(\mu_{i}+\Psi_{i}\right)\right)=\theta_{i} \Delta c_{i} \quad \text { in } Q_{T}:=(0, T) \times \Omega .
$$

Keywords and phrases. two-phase flow, degenerate Cahn-Hilliard system, finite volumes, convergence.

1 Inria, Univ. Lille, CNRS, UMR 8524 - Laboratoire Paul Painlevé, 59000 Lille, France.

2 CMAP, École polytechnique, CNRS, I.P. Paris, 91128 Palaiseau, France.

${ }^{*}$ Corresponding author: clement.cances@inria.fr 
In the above equation, $\eta_{i}>0$ denotes the viscosity of the phase $i, \mu_{i}$ is its chemical potential (which is one of the unknown of the problem), while $\Psi_{i} \in H^{1}(\Omega)$ is a given external potential acting on phase $i$ that is assumed be independent on time for simplicity. For $\Psi_{i}$, one can typically think about gravity, that is $\Psi_{i}(\boldsymbol{x})=-\varrho_{i} \mathbf{g} \cdot \boldsymbol{x}$ with $\varrho_{i}$ the density of phase $i$ and $\mathbf{g}$ the gravitational vector. The coefficient $\theta_{i} \geq 0$ is a given parameter quantifying the thermal agitation of phase $i$. The limit case $\theta_{i}=0$ is called the deep-quench limit in the Cahn-Hilliard literature.

The difference of the phase chemical potentials is given by the following expression

$$
\mu_{1}-\mu_{2}=-\alpha \Delta c_{1}+\kappa\left(1-2 c_{1}\right) \text { in } Q_{T},
$$

where $\alpha>0$ and $\kappa>0$ are given coefficients governing the characteristic size of the transition layers between patches of pure phases $\left\{c_{1}=0\right\}$ and $\left\{c_{1}=1\right\}$. Typically, $\alpha$ is assumed to be small in comparison to $\kappa$. Equation (1.3) is complemented by homogeneous Neumann boundary conditions

$$
\boldsymbol{\nabla} c_{i} \cdot \boldsymbol{n}=0 \quad \text { on }(0, T) \times \partial \Omega
$$

whereas (1.2) is complemented by no-flux boundary conditions

$$
\frac{c_{i}}{\eta_{i}} \boldsymbol{\nabla}\left(\mu_{i}+\Psi_{i}\right) \cdot \boldsymbol{n}=0 \quad \text { on }(0, T) \times \partial \Omega .
$$

Up to now, the chemical potentials are defined up to a common constant. This degree of freedom is fixed by imposing a zero mean condition on the mean chemical potential $\bar{\mu}$, i.e.,

$$
\int_{\Omega} \bar{\mu}(t, \boldsymbol{x}) \mathrm{d} \boldsymbol{x}=0, \quad \forall t \geq 0, \quad \text { where } \bar{\mu}=c_{1} \mu_{1}+c_{2} \mu_{2} .
$$

Finally to close the system, we impose an initial condition $\boldsymbol{c}^{0}=\left(c_{1}^{0}, c_{2}^{0}\right)$ on the volume fractions by setting

$$
c_{\left.i\right|_{t=0}}=c_{i}^{0} \quad \text { in } \Omega \text {. }
$$

The initial profiles $c_{i}^{0} \in H^{1}(\Omega)$ are assumed to be nonnegative with $c_{1}^{0}+c_{2}^{0}=1$ in $\Omega$, and we assume that both phases are present at initial time, i.e.,

$$
\int_{\Omega} c_{i}^{0} \mathrm{~d} \boldsymbol{x}>0, \quad i \in\{1,2\} .
$$

\subsection{Fundamental estimates and weak solutions}

As a preliminary to the study of the numerical scheme, we derive formally at the continuous level some a priori estimates. Their transposition at the discrete level will be key in the numerical analysis to be proposed in what follows. Equation (1.2) can be rewritten under the form

$$
\partial_{t} c_{i}+\nabla \cdot \boldsymbol{F}_{i}=0, \quad \text { with } \quad \boldsymbol{F}_{i}=-\frac{c_{i}}{\eta_{i}} \boldsymbol{\nabla}\left(\mu_{i}+\Psi_{i}+\eta_{i} \theta_{i} \log \left(c_{i}\right)\right) .
$$

In view of the boundary conditions (1.4) and (1.5), this ensures that the volume occupied by each phase is preserved along time, namely

$$
\int_{\Omega} c_{i}(t, \boldsymbol{x}) \mathrm{d} \boldsymbol{x}=\int_{\Omega} c_{i}^{0}(\boldsymbol{x}) \mathrm{d} \boldsymbol{x}, \quad \text { for all } t \geq 0
$$

Moreover, it can be shown by testing $(1.2)$ by $-c_{i}^{-}=\min \left(c_{i}, 0\right)$ that $c_{i} \geq 0$ in $(0, T) \times \Omega$. Thanks to the constraint (1.1), this directly provides that

$$
0 \leq c_{i} \leq 1 \quad \text { in }(0, T) \times \Omega .
$$


Multiplying (1.2) by $\mu_{i}+\Psi_{i}+\eta_{i} \theta_{i} \log \left(c_{i}\right)$, integrating over $\Omega$ and summing over $i$ yields

$$
\sum_{i \in\{1,2\}} \int_{\Omega} \partial_{t} c_{i}\left(\mu_{i}+\Psi_{i}+\eta_{i} \theta_{i} \log \left(c_{i}\right)\right) \mathrm{d} \boldsymbol{x}+\mathfrak{D}(\boldsymbol{c}, \boldsymbol{\mu})=0,
$$

where the energy dissipation $\mathfrak{D}(\boldsymbol{c}, \boldsymbol{\mu})$ is given by

$$
\mathfrak{D}(\boldsymbol{c}, \boldsymbol{\mu})=\sum_{i \in\{1,2\}} \int_{\Omega} \frac{c_{i}}{\eta_{i}}\left|\nabla\left(\mu_{i}+\Psi_{i}+\eta_{i} \theta_{i} \log \left(c_{i}\right)\right)\right|^{2} \mathrm{~d} \boldsymbol{x} \geq 0 .
$$

As a consequence of (1.1), $\partial_{t} c_{2}=-\partial_{t} c_{1}$, so that the first term in the previous inequality can be rewritten as

$$
\sum_{i \in\{1,2\}} \int_{\Omega} \partial_{t} c_{i}\left(\mu_{i}+\Psi_{i}+\eta_{i} \theta_{i} \log \left(c_{i}\right)\right)=\int_{\Omega} \partial_{t} c_{1}\left(\mu_{1}-\mu_{2}\right) \mathrm{d} \boldsymbol{x}+\sum_{i \in\{1,2\}} \int_{\Omega} \partial_{t} c_{i}\left(\Psi_{i}+\eta_{i} \theta_{i} \log \left(c_{i}\right)\right) \mathrm{d} \boldsymbol{x} .
$$

The second term in the right-hand side can be rewritten as

$$
\int_{\Omega} \partial_{t} c_{i}\left(\Psi_{i}+\eta_{i} \theta_{i} \log \left(c_{i}\right)\right) \mathrm{d} \boldsymbol{x}=\frac{\mathrm{d}}{\mathrm{d} t} \int_{\Omega} \sum_{i \in\{1,2\}}\left[c_{i} \Psi_{i}+\eta_{i} \theta_{i} H\left(c_{i}\right)\right] \mathrm{d} \boldsymbol{x}
$$

with

$$
H(c)=c \log (c)-c+1 \geq 0, \quad c \geq 0,
$$

while we can make use of (1.3) to rewrite the first term as

$$
\int_{\Omega} \partial_{t} c_{1}\left(\mu_{1}-\mu_{2}\right) \mathrm{d} \boldsymbol{x}=\frac{\mathrm{d}}{\mathrm{d} t} \int_{\Omega}\left(\frac{\alpha}{2}\left|\nabla c_{1}\right|^{2}+\kappa c_{1}\left(1-c_{1}\right)\right) \mathrm{d} \boldsymbol{x} .
$$

Therefore, we obtain the energy/energy dissipation relation

$$
\frac{\mathrm{d}}{\mathrm{d} t} \mathfrak{E}(\boldsymbol{c})+\mathfrak{D}(\boldsymbol{c}, \boldsymbol{\mu})=0 \quad \text { for all } t \geq 0,
$$

where the energy functional $\mathfrak{E}(\boldsymbol{c})$ is defined by

$$
\mathfrak{E}(\boldsymbol{c})=\int_{\Omega}\left(\frac{\alpha}{2}\left|\nabla c_{1}\right|^{2}+\kappa c_{1}\left(1-c_{1}\right)+\sum_{i \in\{1,2\}}\left[c_{i} \Psi_{i}+\eta_{i} \theta_{i} H\left(c_{i}\right)\right]\right) \mathrm{d} \boldsymbol{x} .
$$

A straightforward consequence of (1.11) is that $t \mapsto \mathfrak{E}(\boldsymbol{c}(t))$ is non-increasing along time, and thus that

$$
\mathfrak{E}(\boldsymbol{c}(t))+\int_{0}^{t} \mathfrak{D}(\boldsymbol{c}(\tau), \boldsymbol{\mu}(\tau)) \mathrm{d} \tau=\mathfrak{E}\left(\boldsymbol{c}^{0}\right)<\infty \quad \text { for all } t \geq 0 .
$$

We deduce from previous inequality that the energy is bounded, hence a $L^{\infty}\left((0, T) ; H^{1}(\Omega)\right)$ estimate on $c_{i}$.

The energy/energy dissipation estimate (1.11) is not sufficient to carry out our mathematical study since it only provides a weighted estimate on the chemical potentials

$$
\sum_{i \in\{1,2\}} \iint_{Q_{T}} c_{i}\left|\nabla \mu_{i}\right|^{2} \mathrm{~d} \boldsymbol{x} \mathrm{d} t \leq C
$$

In order to bypass this difficulty, one needs to quantify the production of mixing entropy. Let us multiply (1.2) by $\eta_{i} \log \left(c_{i}\right)$, integrate over $Q_{T}$ and sum over $i \in\{1,2\}$, which using (1.1) leads to 


$$
\begin{aligned}
\sum_{i \in\{1,2\}} \int_{\Omega} \eta_{i}\left(H\left(c_{i}(T, \cdot)\right)-H\left(c_{i}^{0}\right)\right) \mathrm{d} \boldsymbol{x} & +\sum_{i \in\{1,2\}} \iint_{Q_{T}} \boldsymbol{\nabla} c_{i} \cdot \nabla \Psi_{i} \mathrm{~d} \boldsymbol{x} \mathrm{d} t \\
& +\sum_{i \in\{1,2\}} \theta_{i} \eta_{i} \iint_{Q_{T}} c_{i}\left|\nabla \log \left(c_{i}\right)\right|^{2} \mathrm{~d} \boldsymbol{x} \mathrm{d} t+\iint_{Q_{T}} \boldsymbol{\nabla} c_{1} \cdot \boldsymbol{\nabla}\left(\mu_{1}-\mu_{2}\right) \mathrm{d} \boldsymbol{x} \mathrm{d} t=0 .
\end{aligned}
$$

The first two terms can be bounded thanks to the $L^{\infty}\left(Q_{T}\right)$ and $L^{\infty}\left((0, T) ; H^{1}(\Omega)\right)$ estimates on $c_{i}$. For the last term of the left-hand side, one makes use of (1.3) and (1.4) to rewrite it as

$$
\begin{aligned}
\iint_{Q_{T}} \boldsymbol{\nabla} c_{1} \cdot \nabla\left(\mu_{1}-\mu_{2}\right) \mathrm{d} \boldsymbol{x} \mathrm{d} t & =\iint_{Q_{T}}\left(-\Delta c_{1}\right)\left(-\alpha \Delta c_{1}+\kappa\left(1-2 c_{1}\right)\right) \mathrm{d} \boldsymbol{x} \mathrm{d} t \\
& \geq \frac{\alpha}{2} \iint_{Q_{T}}\left|\Delta c_{1}\right|^{2} \mathrm{~d} \boldsymbol{x} \mathrm{d} t-\frac{\kappa}{2 \alpha} \iint_{Q_{T}}\left(1-2 c_{1}\right)^{2} \mathrm{~d} \boldsymbol{x} \mathrm{d} t .
\end{aligned}
$$

The $L^{\infty}\left(Q_{T}\right)$ estimate on $c_{1}$ shows that the last term of the right-hand side is bounded. At the end of the day, since $c_{i}\left|\nabla \log \left(c_{i}\right)\right|^{2}=4\left|\nabla \sqrt{c_{i}}\right|^{2}$, one gets

$$
\frac{\alpha}{2} \iint_{Q_{T}}\left|\Delta c_{1}\right|^{2} \mathrm{~d} \boldsymbol{x} \mathrm{d} t+\sum_{i \in\{1,2\}} 4 \theta_{i} \eta_{i} \iint_{Q_{T}}\left|\nabla \sqrt{c_{i}}\right|^{2} \mathrm{~d} \boldsymbol{x} \mathrm{d} t \leq C .
$$

Combining this estimate with relation (1.3), we obtain a $L^{2}\left(Q_{T}\right)$ estimate on $\mu_{1}-\mu_{2}$.

The last step aims at obtaining an $L^{2}\left(Q_{T}\right)$ bound on each $\mu_{i}$ independently. The definition (1.6) of $\bar{\mu}$ yields

$$
\nabla \bar{\mu}=\left(\mu_{1}-\mu_{2}\right) \nabla c_{1}+\sum_{i \in\{1,2\}} c_{i} \nabla \mu_{i} .
$$

The first term is in $L^{2}\left((0, T) ; L^{1}(\Omega)\right)$ as the product of an element of $L^{2}\left(Q_{T}\right)$ with an element of $L^{\infty}\left((0, T) ; L^{2}(\Omega)\right)$, while the second term is in $L^{2}\left(Q_{T}\right)$ since $0 \leq c_{i} \leq 1$ and thanks to (1.14). As a consequence, $\nabla \bar{\mu}$ is bounded in $L^{2}\left((0, T) ; L^{1}(\Omega)\right)$. Making use of the Poincaré-Sobolev estimate (recall that $\bar{\mu}$ has zero mean for all time, cf. (1.6), and that $\Omega \subset \mathbb{R}^{2}$ ), we obtain that $\bar{\mu}$ is bounded in $L^{2}\left(Q_{T}\right)$. To get the desired $L^{2}\left(Q_{T}\right)$ estimate on $\mu_{1}$, it only remains to check that

$$
\mu_{1}=\left(c_{1}+c_{2}\right) \mu_{1}=\bar{\mu}-c_{2}\left(\mu_{1}-\mu_{2}\right)
$$

belongs to $L^{2}\left(Q_{T}\right)$ thanks to the $L^{2}\left(Q_{T}\right)$ estimates on $\bar{\mu}$ and $\mu_{1}-\mu_{2}$ together with $0 \leq c_{2} \leq 1$.

The interest of the above formal calculations is twofold. First, our scheme has been designed so that all these calculations can be transposed to the discrete setting. The corresponding a priori estimates will be at the basis of the numerical analysis proposed in this paper. Second, these estimates provide enough regularity on the solution to give a proper notion of weak solution to the problem.

Definition 1.1. $(\boldsymbol{c}, \boldsymbol{\mu})$ is said to be a weak solution to the problem (1.1)-(1.7) if

- $c_{i} \in L^{\infty}\left(Q_{T}\right) \cap L^{\infty}\left((0, T) ; H^{1}(\Omega)\right)$ with $c_{i} \geq 0$ and $c_{1}+c_{2}=1$ a.e. in $Q_{T}$;

- $\mu_{i} \in L^{2}\left(Q_{T}\right)$ with $c_{i} \nabla \mu_{i} \in L^{2}\left(Q_{T}\right)$ and $\int_{\Omega} \bar{\mu}(t, \boldsymbol{x}) \mathrm{d} \boldsymbol{x}=0$ for a.e. $t \in(0, T)$;

- For all $\varphi \in C_{c}^{\infty}([0, T) \times \bar{\Omega})$, there holds

$$
\iint_{Q_{T}} c_{i} \partial_{t} \varphi \mathrm{d} \boldsymbol{x} \mathrm{d} t+\int c_{\Omega}^{0} \varphi(0, \cdot) \mathrm{d} \boldsymbol{x}-\iint_{Q_{T}}\left(\frac{c_{i}}{\eta_{i}} \nabla\left(\mu_{i}+\Psi_{i}\right)+\theta_{i} \boldsymbol{\nabla} c_{i}\right) \cdot \boldsymbol{\nabla} \varphi \mathrm{d} \boldsymbol{x} \mathrm{d} t=0
$$

as well as

$$
\iint_{Q_{T}}\left(\mu_{1}-\mu_{2}\right) \varphi \mathrm{d} \boldsymbol{x} \mathrm{d} t=\iint_{Q_{T}}\left[\alpha \boldsymbol{\nabla} c_{1} \cdot \nabla \varphi+\kappa\left(1-2 c_{1}\right) \varphi\right] \mathrm{d} \boldsymbol{x} \mathrm{d} t
$$


The existence of a weak solution has been established in [8] by showing the convergence of a minimizing movement scheme à la Jordan et al. [23]. Note that in [8], the case of a convex three-dimensional domain $\Omega$ is also addressed, but it relies on the fact that the $L^{2}\left(Q_{T}\right)$ estimate on $\Delta c_{1}$ yields a $L^{2}\left((0, T) ; H^{2}(\Omega)\right)$ estimate on $c_{i}$ for which we don't have an equivalent at the discrete level. This is why we restrict our attention on the case $\Omega \subset \mathbb{R}^{2}$ (but not necessarily convex) in this paper.

\subsection{Some words about the model}

Before entering the core of the paper, which is devoted to the convergence analysis of a finite volume scheme, let us briefly discuss the model under consideration, and in particular its difference with respect to the usual Cahn-Hilliard system. We refer to $[8,29]$ for a more developed discussions on this purpose. The classical degenerate Cahn-Hilliard equation which is the closest one to our system (1.1)-(1.7) writes

$$
\partial_{t} c_{1}-\nabla \cdot\left(\lambda\left(c_{1}\right) \nabla\left(\mu_{\mathrm{g}}+\Psi_{1}-\Psi_{2}\right)\right)=\Delta r\left(c_{1}\right) \text { in }(0, T) \times \Omega,
$$

where the degenerate mobility is given by $\lambda(c)=\frac{c(1-c)}{\eta_{1}+c\left(\eta_{2}-\eta_{1}\right)}$, the function governing the nonlinear diffusion $r$ is such that $r^{\prime}(c)=\lambda(c)\left(\frac{\theta_{1} \eta_{1}}{c}+\frac{\theta_{2} \eta_{2}}{1-c}\right)$, and the generalized chemical potential

$$
\mu_{\mathrm{g}}=\mu_{1}-\mu_{2}=-\alpha \Delta c_{1}+\kappa\left(1-2 c_{1}\right) \quad \text { in }(0, T) \times \Omega .
$$

This system has to be completed with one initial condition on $c_{1}^{0}$ and boundary conditions

$$
-\lambda\left(c_{1}\right) \boldsymbol{\nabla}\left(\mu_{\mathrm{g}}+\Psi_{1}-\Psi_{2}\right) \cdot \boldsymbol{n}=0 \quad \text { and } \quad \boldsymbol{\nabla} c_{1} \cdot \boldsymbol{n}=0 \quad \text { on }(0, T) \times \partial \Omega .
$$

The existence of a solution to this problem has been addressed in [14,25].

Let us come back to our system (1.1)-(1.7). Denote the total flux by $\boldsymbol{F}_{\text {tot }}=\boldsymbol{F}_{1}+\boldsymbol{F}_{2}$, then summing (1.9) over $i \in\{1,2\}$ yields

$$
\boldsymbol{\nabla} \cdot \boldsymbol{F}_{\mathrm{tot}}=-\partial_{t}\left(c_{1}+c_{2}\right)=0 \quad \text { in }(0, T) \times \Omega
$$

owing to (1.1). After some elementary calculations, the conservation (1.2) for the phase 1 rewrites

$$
\partial_{t} c_{1}+\boldsymbol{\nabla} \cdot\left(f\left(c_{1}\right) \boldsymbol{F}_{\text {tot }}-\lambda\left(c_{1}\right) \boldsymbol{\nabla}\left(\mu_{\mathrm{g}}+\Psi_{1}-\Psi_{2}\right)\right)=\Delta r\left(c_{1}\right) \quad \text { in }(0, T) \times \Omega,
$$

where $f(c)=\frac{\eta_{2} c}{\eta_{1}+\left(\eta_{2}-\eta_{1}\right) c}$. The equation (1.21) differs from (1.18) by the addition of a nonlinear transport term driven by a divergence free vector field. Both systems can be reinterpreted as Wasserstein-type gradient flows [1] of the energy $\mathfrak{E}(\boldsymbol{c})$ for different geometries:

- the Wasserstein distance with quadratic cost with the constraint $\boldsymbol{F}_{\text {tot }}=\mathbf{0}$ for the classical Cahn-Hilliard system, $c f .[25]$;

- the Wasserstein distance with quadratic cost with the less stringent constraint $\boldsymbol{\nabla} \cdot \boldsymbol{F}_{\text {tot }}=0$ for the system (1.1)-(1.7), cf. [8,29].

The additional degree of freedom $\boldsymbol{F}_{\text {tot }}$ allows the energy $\mathfrak{E}(\boldsymbol{c})$ to decrease faster along the trajectories, as highlighted in [8]. Finally, let us point the recent contribution [7] where the convergence of a minimizing movement scheme is addressed for a closely related model where the Cahn-Hilliard energy is replaced by the singular de Gennes-Flory-Higgins energy.

\section{Finite volume approximation AND MAIN RESUlts}

Prior to presenting the scheme and stating our main results, that are the existence of a discrete solution to the scheme and the convergence of the corresponding approximate solutions towards a weak solution to the problem (1.1)-(1.7), we introduce some notations and requirements concerning the mesh. 


\section{1. (Super)-admissible mesh of $\Omega$ and time discretization}

Let us first give a definition of what we call an admissible mesh.

Definition 2.1. An admissible mesh of $\Omega$ is a triplet $\left(\mathcal{T}, \mathcal{E},\left(\boldsymbol{x}_{K}\right)_{K \in \mathcal{T}}\right)$ such that the following conditions are fulfilled.

(i) Each control volume (or cell) $K \in \mathcal{T}$ is non-empty, open, polygonal and convex. We assume that

$$
K \cap L=\emptyset \quad \text { if } K, L \in \mathcal{T} \text { with } K \neq L, \quad \text { while } \bigcup_{K \in \mathcal{T}} \bar{K}=\bar{\Omega} .
$$

We denote by $m_{K}$ the 2-dimensional Lebesgue measure of $K$.

(ii) Each edge $\sigma \in \mathcal{E}$ is closed and is contained in a hyperplane of $\mathbb{R}^{2}$, with positive 1-dimensional Hausdorff (or Lebesgue) measure denoted by $m_{\sigma}=\mathcal{H}^{1}(\sigma)>0$. We assume that $\mathcal{H}^{1}\left(\sigma \cap \sigma^{\prime}\right)=0$ for $\sigma, \sigma^{\prime} \in \mathcal{E}$ unless $\sigma^{\prime}=\sigma$. For all $K \in \mathcal{T}$, we assume that there exists a subset $\mathcal{E}_{K}$ of $\mathcal{E}$ such that $\partial K=\bigcup_{\sigma \in \mathcal{E}_{K}} \sigma$. Moreover, we suppose that $\bigcup_{K \in \mathcal{T}} \mathcal{E}_{K}=\mathcal{E}$. Given two distinct control volumes $K, L \in \mathcal{T}$, the intersection $\bar{K} \cap \bar{L}$ either reduces to a single edge $\sigma \in \mathcal{E}$ denoted by $K \mid L$, or its 1-dimensional Hausdorff measure is 0 .

(iii) The cell-centers $\left(\boldsymbol{x}_{K}\right)_{K \in \mathcal{T}}$ are pairwise distinct with $\boldsymbol{x}_{K} \in K$, and are such that, if $K, L \in \mathcal{T}$ share an edge $K \mid L$, then the vector $\boldsymbol{n}_{K L}=\frac{\boldsymbol{x}_{L}-\boldsymbol{x}_{K}}{\left|\boldsymbol{x}_{K}-\boldsymbol{x}_{L}\right|}$ is orthogonal to $K \mid L$.

(iv) Given two cells $K, L \in \mathcal{T}$ sharing an edge $\sigma=K \mid L$, we assume that the straight line joining $\boldsymbol{x}_{K}$ and $\boldsymbol{x}_{L}$ crosses the edge $\sigma$ in its midpoint $\boldsymbol{x}_{\sigma}$.

Let us introduce some additional notations, some of them being depicted on Figure 1 . The size of the mesh $\mathcal{T}$ (which is intended to tend to 0 in the convergence proof) is defined by $h_{\mathcal{T}}=\max _{K \in \mathcal{T}} h_{K}$, with $h_{K}=\operatorname{diam}(K)$. Given two neighboring cells $K, L \in \mathcal{T}$ sharing an edge $\sigma=K \mid L$, we denote by $d_{\sigma}=\left|\boldsymbol{x}_{K}-\boldsymbol{x}_{L}\right|$ whereas $d_{K \sigma}=\left|\boldsymbol{x}_{K}-\boldsymbol{x}_{\sigma}\right| \leq d_{\sigma}$. The transmissivities $\tau_{\sigma}$ and $\tau_{K \sigma}$ of the edge $\sigma$ are respectively defined by $\tau_{\sigma}=\frac{m_{\sigma}}{d_{\sigma}}$ and $\tau_{K \sigma}=\frac{m_{\sigma}}{d_{K \sigma}}$. The diamond $D_{\sigma}$ and half diamond $D_{K \sigma}$ cells are defined as the convex hulls of $\left\{\boldsymbol{x}_{K}, \boldsymbol{x}_{L}, \sigma\right\}$ and $\left\{\boldsymbol{x}_{K}, \sigma\right\}$ respectively. Denoting by $m_{D_{\sigma}}$ (resp. $m_{D_{K \sigma}}$ ) the 2-dimensional Lebesgue measure of $D_{\sigma}$ (resp. $D_{K \sigma}$ ), we will use many time the following elementary geometric properties: $d_{\sigma} m_{\sigma}=2 m_{D_{\sigma}}$ and $d_{K \sigma} m_{\sigma}=2 m_{D_{K \sigma}}$. We also denote by $\mathcal{E}_{K \text {,int }}$ the subset of $\mathcal{E}_{K}$ made of the internal edges $\sigma$ such that there exists $L \in \mathcal{T}$ such that $\sigma=K \mid L$, and by $\mathcal{E}_{\text {int }}=\bigcup_{K \in \mathcal{T}} \mathcal{E}_{K, \text { int }}$.

Even though this is absolutely not necessary, we choose to restrict our attention to the case of uniform time discretizations in the mathematical proofs in order to reduce the amount of notations. In what follows, we set $\Delta t=T / N$ and $t^{n}=n \Delta t$ for $n \in\{0, \ldots, N\}$. The integer $N$ is intended to be large and even to tend to $+\infty$ in the convergence proof.

Remark 2.2. Condition (iv) above enforces an additional restriction with respect to the classical definition of finite volumes meshes with orthogonality condition (iii). Meshes satisfying this condition in addition to the more classical assumptions (i)-(iii) is called super-admissible following the terminology introduced in [17]. It is for instance satisfied by cartesian grids or by acute triangulations. However, (iv) is in general not satisfied by Voronoï meshes. This condition appears for technical reasons related to the construction of a strongly convergent SUSHI discrete gradient [17], see Proposition 4.2 later on. On the other hand, this condition was recently pushed forward in [20] to show the consistency of the discrete optimal transportation geometry [26] hidden behind our work with the continuous optimal transportation geometry [31] in which our system (1.1)-(1.7) has a gradient flow structure [8].

\subsection{A two-point flux approximation finite volume scheme}

The scheme we propose is a cell-centered scheme based on two-point flux approximation (TPFA) finite volumes. At each time step $n \in\{1, \ldots, N\}$, then unknowns are located at the centers $\boldsymbol{x}_{K}$ of the cells $K \in \mathcal{T}$. Given discrete volume fractions $\boldsymbol{c}^{n-1}=\left(c_{1, K}^{n-1}, c_{2, K}^{n-1}\right)_{K \in \mathcal{T}}$ at time $t^{n-1}$, we look for updated volume fractions 

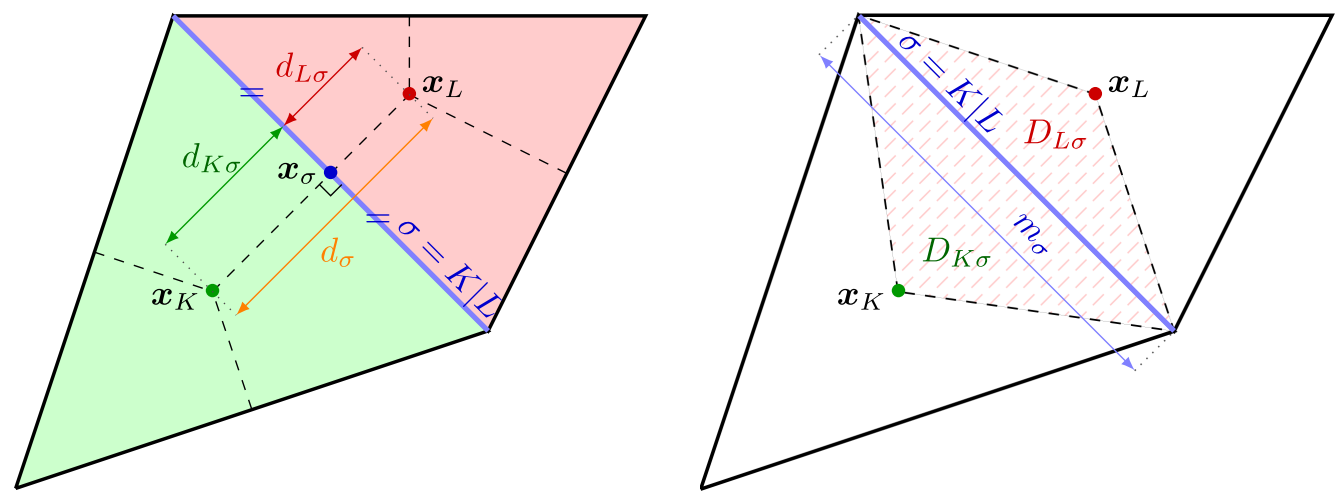

FiguRE 1. Illustration of an admissible mesh in the sense of Definition 2.1. Each point $\boldsymbol{x}_{K}$ belongs to the cell $K$ for $K \in \mathcal{T}$. For any $\sigma=K \mid L$, the segment $\left[\boldsymbol{x}_{K}, \boldsymbol{x}_{L}\right]$ intersects $\sigma$ at its midpoint $\boldsymbol{x}_{\sigma}$ in an orthogonal way. This properties hold for meshes made of triangles with acute angles if $\boldsymbol{x}_{K}$ is chosen as the center of the circumcircle of the triangle $K$. On the right figure, the dashed area is the diamond cell $D_{\sigma}$ corresponding to the edge $\sigma=K \mid L$. It is made of $D_{K, \sigma}=D_{\sigma} \cap K$ (in green), $D_{L \sigma}=D_{\sigma} \cap L$ (in red), and of the edge $\sigma=K \mid L$ (in blue), the length of which is equal to $m_{\sigma}$.

$\boldsymbol{c}^{n}=\left(c_{1, K}^{n}, c_{2, K}^{n}\right)_{K \in \mathcal{T}}$ and chemical potentials $\boldsymbol{\mu}^{n}=\left(\mu_{1, K}^{n}, \mu_{2, K}^{n}\right)_{K \in \mathcal{T}}$ at time $t^{n}$ that are expected to approximate the mean values on $K$ of their continuous counterparts $\boldsymbol{c}\left(t^{n}\right)$ and $\boldsymbol{\mu}\left(t^{n}\right)$. At time $t=0$, we initialize the procedure by setting

$$
c_{i, K}^{0}=\frac{1}{m_{K}} \int_{K} c_{i}^{0} \mathrm{~d} \boldsymbol{x}, \quad \forall K \in \mathcal{T}, i \in\{1,2\} .
$$

As highlighted in the formal calculations presented in Section 1.2, the analysis requires the use of the logarithm of the volume fractions. To this end, the volume fractions $c_{i, K}^{n}$ have to be strictly positive for $n \geq 1$. To ensure this property, some thermal diffusion is needed, see Lemma 3.2. In the case where $\theta_{i}=0$, then one needs to introduce a small amount of numerical diffusion by setting

$$
\theta_{i, \mathcal{T}}=\max \left(\theta_{i}, \rho h_{\mathcal{T}}\right)>0, \quad i \in\{1,2\},
$$

where $\rho>0$ is a parameter that can be fixed by the user. Equation (1.2) is then discretized into

$$
m_{K} \frac{c_{i, K}^{n}-c_{i, K}^{n-1}}{\Delta t}+\sum_{\substack{\sigma \in \mathcal{E}_{K, \text { int }} \\ \sigma=K \mid L}} \tau_{\sigma}\left[\frac{c_{i, \sigma}^{n}}{\eta_{i}}\left(\mu_{i, K}^{n}+\Psi_{i, K}-\mu_{i, L}^{n}-\Psi_{i, L}\right)+\theta_{i, \mathcal{T}}\left(c_{i, K}^{n}-c_{i, L}^{n}\right)\right]=0
$$

for all $K \in \mathcal{T}$ and $i \in\{1,2\}$. In the above relation, we used the following discretization of the external potential:

$$
\Psi_{i, K}=\frac{1}{m_{K}} \int_{K} \Psi_{i}(\boldsymbol{x}) \mathrm{d} \boldsymbol{x}, \quad \forall K \in \mathcal{T}, i \in\{1,2\} .
$$

Edge values $c_{i, \sigma}^{n}$ of the discrete volume fractions also appear in (2.3). Rather than using upstream values of the volume fractions as in our previous work [9], we make use of a logarithmic mean, i.e.,

$$
c_{i, \sigma}^{n}= \begin{cases}c_{i, K}^{n} & \text { if } c_{i, K}^{n}=c_{i, L}^{n} \geq 0, \\ 0 & \text { if } \min \left(c_{i, K}^{n}, c_{i, L}^{n}\right) \leq 0, \\ \frac{c_{i, K}^{n}-c_{i, L}^{n}}{\log \left(c_{i, K}^{n}\right)-\log \left(c_{i, L}^{n}\right)} & \text { otherwise, }\end{cases}
$$


for all $\sigma=K \mid L \in \mathcal{E}_{\text {int }}$ and $i \in\{1,2\}$. This particular choice of the edge value is closely related to the one introduced in the early work [22] for the approximation of the thin film equation, and fits with the one suggested in [26,28] and used in a closely related context to ours in [27]. Equation (1.3) is discretized into

$$
\mu_{1, K}^{n}-\mu_{2, K}^{n}=\frac{\alpha}{m_{K}} \sum_{\substack{\sigma \in \mathcal{E}_{K, \text { int }} \\ \sigma=K \mid L}} \tau_{\sigma}\left(c_{1, K}^{n}-c_{1, L}^{n}\right)+\kappa\left(1-2 c_{1, K}^{n-1}\right), \quad \forall K \in \mathcal{T} .
$$

Note that the repulsive term (second in the right-hand side) is discretized in an explicit way for stability issues that will appear clearly later on. The constraint (1.1) is discretized in a straightforward way by imposing

$$
c_{1, K}^{n}+c_{2, K}^{n}=1, \quad \forall K \in \mathcal{T} .
$$

The last equation to be transposed in the discrete setting is (1.6), which is translated into

$$
\sum_{K \in \mathcal{T}} m_{K} \bar{\mu}_{K}^{n}=0, \quad \text { where } \bar{\mu}_{K}^{n}=c_{1, K}^{n} \mu_{1, K}^{n}+c_{2, K}^{n} \mu_{2, K}^{n} .
$$

\subsection{Main results}

Before addressing the convergence of the scheme, we focus first on the case of a fixed mesh and time discretization. The scheme (2.3)-(2.7) yields a nonlinear system on $\left(\boldsymbol{c}^{n}, \boldsymbol{\mu}^{n}\right)$. The existence of a solution to this nonlinear system is far from being obvious. The existence of such a solution and some important properties of the discrete solution mimicking the properties highlighted in Section 1.2 are gathered in the first theorem of this paper.

Theorem 2.3. Assume that the inverse CFL condition (3.18) is fulfilled, then there exists (at least) one solution $\left(\boldsymbol{c}^{n}, \boldsymbol{\mu}^{n}\right)_{n \geq 1}$ to the scheme (2.3)-(2.7). Moreover, this solution satisfies the following properties:

(i) mass conservation:

$$
\sum_{K \in \mathcal{T}} m_{K} c_{i, K}^{n}=\int_{\Omega} c_{i}^{0} \mathrm{~d} \boldsymbol{x}, \quad n \geq 0, i \in\{1,2\}
$$

(ii) positivity:

$$
0<c_{i, K}^{n}<1, \quad K \in \mathcal{T}, i \in\{1,2\}, n \geq 1
$$

(iii) energy decay:

$$
\frac{\mathfrak{E}_{\mathcal{T}}\left(\boldsymbol{c}^{n}\right)-\mathfrak{E}_{\mathcal{T}}\left(\boldsymbol{c}^{n-1}\right)}{\Delta t}+\mathfrak{D}\left(\boldsymbol{c}^{n}, \boldsymbol{\mu}^{n}\right) \leq 0, \quad \forall n \geq 1,
$$

where the discrete energy $\mathfrak{E}\left(\boldsymbol{c}^{n}\right)$ is defined by

$$
\begin{aligned}
\mathfrak{E}_{\mathcal{T}}\left(\boldsymbol{c}^{n}\right)= & \frac{\alpha}{2} \sum_{\substack{\sigma \in \mathcal{E}_{\text {int }} \\
\sigma=K \mid L}} \tau_{\sigma}\left(c_{1, K}^{n}-c_{1, L}^{n}\right)^{2} \\
& +\sum_{K \in \mathcal{T}} m_{K}\left\{\kappa c_{1, K}^{n} c_{2, K}^{n}+\sum_{i=\{1,2\}}\left(c_{i, K}^{n} \Psi_{i, K}+\theta_{i, \mathcal{T}} \eta_{i} H\left(c_{i, K}^{n}\right)\right)\right\},
\end{aligned}
$$

where $H$ is given by (1.10), and the discrete dissipation is defined by

$$
\mathfrak{D}_{\mathcal{T}}\left(\boldsymbol{c}^{n}, \boldsymbol{\mu}^{n}\right)=\sum_{i \in\{1,2\}} \sum_{\substack{\sigma \in \mathcal{E}_{\text {int }} \\ \sigma=K \mid L}} \tau_{\sigma} \frac{c_{i, \sigma}^{n}}{\eta_{i}}\left(\mu_{i, K}^{n}+\Psi_{i, K}-\mu_{i, L}^{n}-\Psi_{i, L}+\theta_{i, \mathcal{T}} \eta_{i}\left(\log \left(c_{i, K}^{n}\right)-\log \left(c_{i, L}^{n}\right)\right)\right)^{2} .
$$


The existence of a solution to the scheme for each time step allows to reconstruct an approximate solution $\left(\boldsymbol{c}_{\mathcal{T}, \Delta t}, \boldsymbol{\mu}_{\mathcal{T}, \Delta t}\right)$ with $\boldsymbol{c}_{\mathcal{T}, \Delta t}=\left(c_{1, \mathcal{T}, \Delta t}, c_{2, \mathcal{T}, \Delta t}\right)$ and $\boldsymbol{\mu}_{\mathcal{T}, \Delta t}=\left(\mu_{1, \mathcal{T}, \Delta t}, \mu_{2, \mathcal{T}, \Delta t}\right)$ by setting

$$
c_{i, \mathcal{T}, \Delta t}(t, \boldsymbol{x})=c_{i, K}^{n} \quad \text { and } \quad \mu_{i, \mathcal{T}, \Delta t}(t, \boldsymbol{x})=\mu_{i, K}^{n} \quad \text { if }(t, \boldsymbol{x}) \in\left(t^{n-1}, t^{n}\right] \times K, \quad i \in\{1,2\} .
$$

The approximate solutions are expected to approximate the continuous solution to (1.1)-(1.7). Our second theorem gives a mathematical foundation to this statement. It requires the introduction of a suitable sequence of discretizations of $Q_{T}$. In what follows, we denote by $\left(\mathcal{T}_{m}, \mathcal{E}_{m},\left(\boldsymbol{x}_{K}\right)_{K \in \mathcal{T}_{m}}\right)_{m>1}$ a sequence of admissible meshes of $\Omega$ is the sense of Definition 2.1. We assume that $h_{\mathcal{T}_{m}}$ tends to 0 as $m \rightarrow \infty$ as well as the following regularity requirements:

- shape regularity of the cells: there exists a finite $\zeta>1$ such that

$$
d_{\sigma} \leq \zeta d_{K \sigma}, \quad \forall m \geq 1, \forall K \in \mathcal{T}_{m}, \forall \sigma \in \mathcal{E}_{K, \text { int }, m},
$$

and such that

$$
m_{K} \geq \frac{1}{\zeta}\left(h_{K}\right)^{2}, \quad \forall m \geq 1, \forall K \in \mathcal{T}_{m} ;
$$

- boundedness of the number of edges per element: there exists $\ell^{\star} \geq 3$ such that

$$
\# \mathcal{E}_{K} \leq \ell^{\star}, \quad \forall m \geq 1, \forall K \in \mathcal{T}_{m}
$$

- control on the transmissivities: there exist $\tau^{\star}, \tau_{\star} \geq 0$ such that

$$
\tau^{\star} \geq \tau_{\sigma} \geq \tau_{\star}>0, \quad \forall m \geq 1, \forall \sigma \in \mathcal{E}_{\text {int }, m} .
$$

The combination of a sequence $\left(\mathcal{T}_{m}, \mathcal{E}_{m},\left(\boldsymbol{x}_{K}\right)_{K \in \mathcal{T}_{m}}\right)_{m>1}$ fulfilling (2.11)-(2.14) together with a time step $\Delta t_{m}=$ $T / N_{m}$ and $N_{m} \rightarrow+\infty$ as $m \rightarrow \infty$ is said to be a regular discretization of $Q_{T}$ if it moreover satisfies the inverse CFL condition (3.18).

Theorem 2.4. Let $\left(\mathcal{T}_{m}, \mathcal{E}_{m},\left(\boldsymbol{x}_{K}\right)_{K \in \mathcal{T}_{m}}, \Delta t_{m}\right)_{m \geq 1}$ be a sequence of regular discretizations of $Q_{T}$, and let $\left(\boldsymbol{c}_{\mathcal{T}_{m}, \Delta t_{m}}, \boldsymbol{\mu}_{\mathcal{T}_{m}, \Delta t_{m}}\right)_{m \geq 1}$ be a corresponding sequence of approximate solutions. Then there exists a weak solution $(\boldsymbol{c}, \boldsymbol{\mu})$ to $(1.1)-(1.7)$ in the sense of Definition 1.1 such that, up to a subsequence,

$$
c_{i, \mathcal{T}_{m}, \Delta t_{m}} \underset{m \rightarrow \infty}{\longrightarrow} c_{i} \quad \text { a.e. in } Q_{T} \quad \text { and } \quad \mu_{i, \mathcal{T}_{m}, \Delta t_{m}} \underset{m \rightarrow \infty}{\longrightarrow} \mu_{i} \quad \text { weakly in } L^{2}\left(Q_{T}\right) .
$$

The convergence properties stated in Theorem 2.4 are weaker than what is practically proved in the paper. The statement of optimal convergence properties would require the introduction of additional material that we postpone to the proof in order to optimize the readability of the paper.

The remaining of the paper is organized as follows. In Section 3, we work at fixed mesh and time step. We derive some a priori estimates and show the existence of (at least) one solution to the scheme thanks to a topological degree argument. Next in Section 4, we show thanks to compactness arguments that the approximate solution converge towards a weak solution to the scheme. Finally, we present in Section 5 some numerical simulations.

\section{A priori estimates And EXistence of A Discrete solution}

In Section 3.1, we first derive some a priori estimates on the solutions to the scheme (2.3)-(2.7). These estimates will be at the basis of the existence proof for a discrete solution to the scheme in Section 3.2, but also of the convergence proof carried out in Section 4. 


\subsection{A priori estimates}

This section is devoted to the derivation to all the a priori estimates needed in the numerical analysis of the scheme. The first of them is the global conservation of mass, which is a consequence of the local conservativity of the scheme.

Lemma 3.1. For all $n \geq 0$ and $i \in\{1,2\}$, there holds

$$
\sum_{K \in \mathcal{T}} m_{K} c_{i, K}^{n}=\int_{\Omega} c_{i}^{0} \mathrm{~d} \boldsymbol{x}>0 .
$$

Proof. Summing (2.3) over $K \in \mathcal{T}$ and using the conservativity of the scheme leads to

$$
\sum_{K \in \mathcal{T}} m_{K} c_{i, K}^{n}=\sum_{K \in \mathcal{T}} m_{K} c_{i, K}^{n-1}, \quad i \in\{1,2\}, n \in\{1, \ldots, N\}
$$

A straightforward induction and the definition (2.1) of $c_{i, K}^{0}$ then provides (3.1).

Our second lemma shows that the volume fractions are positive.

Lemma 3.2. Let $n \geq 1$, and let $\left(\boldsymbol{c}^{n}, \boldsymbol{\mu}^{n}\right)$ be a solution to the scheme (2.3)-(2.7), then

$$
0<c_{i, K}^{n}<1, \quad \forall K \in \mathcal{T}, \forall n \geq 1, \forall i \in\{1,2\} .
$$

Proof. Assume by induction that $0 \leq c_{i, K}^{n-1}$ and $\sum_{K} m_{K} c_{i, K}^{n-1}>0$ (the later having been established for all $n \geq 1$ in Lem. 3.1), and suppose for contradiction that

$$
c_{i, K}^{n}=\min _{L \in \mathcal{T}} c_{i, L}^{n} \leq 0
$$

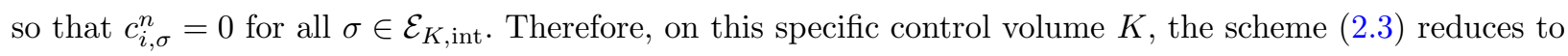

$$
m_{K} \frac{c_{i, K}^{n}-c_{i, K}^{n-1}}{\Delta t}+\theta_{i, \mathcal{T}} \sum_{\substack{\sigma \in \mathcal{E}_{K, \text { int }} \\ \sigma=K \mid L}} \tau_{\sigma}\left(c_{i, K}^{n}-c_{i, L}^{n}\right)=0 .
$$

The left-hand side is nonpositive, and even negative unless $c_{i, L}^{n}=c_{i, K}^{n} \leq 0$ for all the neighbouring cells $L$ of $K$. We can thus iterate the argument and show that $c_{i, L}^{n} \leq 0$ for all $L \in \mathcal{T}$, which provides a contradiction with the property $\sum_{K} m_{K} c_{i, K}^{n}>0$ established in Lemma 3.1.

As a consequence of Lemma 3.2, the quantities $\log \left(c_{i, K}^{n}\right)$ have a sense. They will be used many times along the paper. Our next lemma consists in discrete counterparts of the energy/energy dissipation relations (1.11)-(1.13).

Lemma 3.3. Let $\left(\boldsymbol{c}^{n}, \boldsymbol{\mu}^{n}\right)$ be a solution to the scheme (2.3)-(2.7), then the following discrete energy dissipation relation holds

$$
\frac{\mathfrak{E}_{\mathcal{T}}\left(\boldsymbol{c}^{n}\right)-\mathfrak{E}_{\mathcal{T}}\left(\boldsymbol{c}^{n-1}\right)}{\Delta t}+\mathfrak{D}_{\mathcal{T}}\left(\boldsymbol{c}^{n}, \boldsymbol{\mu}^{n}\right) \leq 0, \quad \forall n \geq 1,
$$

where the discrete energy $\mathfrak{E}_{\mathcal{T}}$ and the discrete dissipation $\mathfrak{D}_{\mathcal{T}}$ are defined by (2.8) and (2.9) respectively.

Proof. Multiplying (2.3) by $\mu_{i, K}^{n}+\Psi_{i, K}+\theta_{i, \mathcal{T}} \eta_{i} \log \left(c_{i, K}^{n}\right)$ and summing over $i \in\{1,2\}$ and $K \in \mathcal{T}$ yields

$$
A_{1}+A_{2}+A_{3}+\mathfrak{D}_{\mathcal{T}}\left(\boldsymbol{c}^{n}, \boldsymbol{\mu}^{n}\right)=0,
$$


where

$$
A_{1}=\sum_{K \in \mathcal{T}} \frac{m_{K}}{\Delta t} \sum_{i \in\{1,2\}}\left(c_{i, K}^{n}-c_{i, K}^{n-1}\right) \mu_{i, K}^{n}, \quad A_{2}=\sum_{K \in \mathcal{T}} \frac{m_{K}}{\Delta t} \sum_{i \in\{1,2\}}\left(c_{i, K}^{n}-c_{i, K}^{n-1}\right) \theta_{i, \mathcal{T}} \eta_{i} \log \left(c_{i, K}^{n}\right),
$$

and

$$
A_{3}=\sum_{K \in \mathcal{T}} \frac{m_{K}}{\Delta t} \sum_{i \in\{1,2\}}\left(c_{i, K}^{n}-c_{i, K}^{n-1}\right) \Psi_{i, K}
$$

It follows from a convexity inequality that

$$
A_{2} \geq \sum_{K \in \mathcal{T}} \frac{m_{K}}{\Delta t} \sum_{i \in\{1,2\}} \theta_{i, \mathcal{T}} \eta_{i}\left(H\left(c_{i, K}^{n}\right)-H\left(c_{i, K}^{n-1}\right)\right) .
$$

Using (2.6) and (2.5), the term $A_{1}$ rewrites

$$
A_{1}=\sum_{K \in \mathcal{T}} \frac{m_{K}}{\Delta t}\left(c_{1, K}^{n}-c_{1, K}^{n-1}\right)\left(\mu_{1, K}^{n}-\mu_{2, K}^{n}\right)=A_{11}+A_{12}
$$

with

$$
\begin{aligned}
& A_{11}=\frac{\alpha}{\Delta t} \sum_{\substack{\sigma \in \mathcal{E}_{\text {int }} \\
\sigma=K \mid L}} \tau_{\sigma}\left(c_{1, K}^{n}-c_{1, L}^{n}\right)\left(c_{1, K}^{n}-c_{1, L}^{n}-c_{1, K}^{n-1}+c_{1, L}^{n-1}\right) \\
& A_{12}=\kappa \sum_{K \in \mathcal{T}} \frac{m_{K}}{\Delta t}\left(1-2 c_{1, K}^{n-1}\right)\left(c_{1, K}^{n}-c_{1, K}^{n-1}\right) .
\end{aligned}
$$

Using again elementary convexity inequalities, one gets that

$$
A_{11} \geq \frac{\alpha}{2 \Delta t} \sum_{\substack{\sigma \in \mathcal{E}_{\text {int }} \\ \sigma=K \mid L}} \tau_{\sigma}\left(\left(c_{1, K}^{n}-c_{1, L}^{n}\right)^{2}-\left(c_{1, K}^{n-1}-c_{1, L}^{n-1}\right)^{2}\right),
$$

and

$$
A_{12} \geq \kappa \sum_{K \in \mathcal{T}} \frac{m_{K}}{\Delta t}\left\{c_{1, K}^{n}\left(1-c_{1, K}^{n}\right)-c_{1, K}^{n-1}\left(1-c_{1, K}^{n-1}\right)\right\} .
$$

The relation (2.6) allows to rewrite the right-hand side of the above inequality, so that

$$
A_{12} \geq \kappa \sum_{K \in \mathcal{T}} \frac{m_{K}}{\Delta t}\left\{c_{1, K}^{n} c_{2, K}^{n}-c_{1, K}^{n-1} c_{2, K}^{n-1}\right\} .
$$

The combination of (3.4)-(3.8) in (3.3) concludes the proof of Lemma 3.3.

The boundedness of the discrete energy $\mathfrak{E}\left(\boldsymbol{c}^{n}\right)$ provides a discrete $L^{\infty}\left((0, T) ; H^{1}(\Omega)\right)$ estimate on the volume fractions, as established in the next corollary.

Corollary 3.4. There exists $C_{1}$ depending only on $\Omega, \alpha, \kappa, \Psi, \theta_{i}, c_{i}^{0}$, and $\zeta$ such that

$$
\sum_{\substack{\sigma \in \mathcal{E}_{\text {int }} \\ \sigma=K \mid L}} \tau_{\sigma}\left(c_{1, K}^{n}-c_{1, L}^{n}\right)^{2} \leq C_{1}
$$


Proof. As a straightforward consequence of Lemma 3.3, the energy is decaying along the time steps, so that

$$
\begin{aligned}
\sum_{\substack{\sigma \in \mathcal{E}_{\text {int }} \\
\sigma=K \mid L}} \tau_{\sigma}\left(c_{1, K}^{n}-c_{1, L}^{n}\right)^{2}+\frac{2}{\alpha} \sum_{i \in\{1,2\}} \sum_{K \in \mathcal{T}} m_{K} c_{i, K}^{n} \Psi_{i, K} \leq \frac{2}{\alpha} \mathfrak{E}_{\mathcal{T}}\left(\boldsymbol{c}^{n}\right) \leq \frac{2}{\alpha} \mathfrak{E}_{\mathcal{T}}\left(\boldsymbol{c}^{0}\right) \\
\quad \leq \sum_{\substack{\sigma \in \mathcal{E}_{\text {int }} \\
\sigma=K \mid L}} \tau_{\sigma}\left(c_{1, K}^{0}-c_{1, L}^{0}\right)^{2}+\frac{2}{\alpha} \sum_{K \in \mathcal{T}} m_{K}\left\{\kappa c_{1, K}^{0} c_{2, K}^{0}+\sum_{i \in\{1,2\}}\left[\Psi_{i, K} c_{i, K}^{0}+\theta_{i, \mathcal{T}} \eta_{i} H\left(c_{i, K}^{0}\right)\right]\right\} .
\end{aligned}
$$

Since $c_{1, K}^{0}+c_{2, K}^{0}=1$, there holds

$$
\sum_{K \in \mathcal{T}} m_{K} \kappa c_{1, K}^{0} c_{2, K}^{0} \leq \frac{\kappa}{4}|\Omega|
$$

Owing to Lemma 9.4 of [16], there exists $C_{2}$ depending only on $\zeta$ such that

$$
\sum_{\substack{\sigma \in \mathcal{E}_{\text {int }} \\ \sigma=K \mid L}} \tau_{\sigma}\left(c_{1, K}^{0}-c_{1, L}^{0}\right)^{2} \leq C_{2} \int_{\Omega}\left|\nabla c_{1}^{0}\right|^{2} \mathrm{~d} \boldsymbol{x}
$$

whereas Jensen's inequality ensures that

$$
\sum_{K \in \mathcal{T}} m_{K} \theta_{i, \mathcal{T}} \eta_{i} H\left(c_{i, K}^{0}\right) \leq \theta_{i, \mathcal{T}} \eta_{i} \int_{\Omega} H\left(c_{i}^{0}\right) \mathrm{d} \boldsymbol{x} \leq \theta_{i, \mathcal{T}} \eta_{i}|\Omega|
$$

Finally, since $0 \leq c_{i, K}^{n} \leq 1$ for $n \geq 0$, we have

$$
\sum_{\substack{\sigma \in \mathcal{E}_{\text {int }} \\ \sigma=K \mid L}} \tau_{\sigma}\left(c_{1, K}^{n}-c_{1, L}^{n}\right)^{2} \leq C_{2} \int_{\Omega}\left|\nabla c_{1}^{0}\right|^{2} \mathrm{~d} \boldsymbol{x}+\frac{|\Omega|}{\alpha}\left(\frac{\kappa}{2}+2 \sum_{i \in\{1,2\}}\left(\theta_{i, \mathcal{T}} \eta_{i}+\frac{2}{|\Omega|}\left\|\Psi_{i}\right\|_{L^{1}(\Omega)}\right)\right) .
$$

Let us now focus on the quantification of the production of mixing entropy at the discrete level. Our next lemma provides a discrete counterpart to Estimate (1.15).

Lemma 3.5. There exists $C_{3}$ depending only on $\Omega, \alpha, \kappa, T, \Psi_{i}, \eta_{i}, \theta_{i}, \zeta$ and $c_{1}^{0}$ such that

$$
\begin{aligned}
\sum_{n=1}^{N} \Delta t \sum_{K \in \mathcal{T}} m_{K}\left(\frac{1}{m_{K}} \sum_{\substack{\sigma \in \mathcal{E}_{K, \text { int }} \\
\sigma=K \mid L}} \tau_{\sigma}\left(c_{1, K}^{n}-c_{1, L}^{n}\right)\right)^{2} & \\
& +\sum_{n=1}^{N} \Delta t \sum_{i \in\{1,2\}} \eta_{i} \theta_{i, \mathcal{T}} \sum_{\substack{\sigma \in \mathcal{E}_{\text {int }} \\
\sigma=K \mid L}} \tau_{\sigma}\left(c_{i, K}^{n}-c_{i, L}^{n}\right)\left(\log \left(c_{i, K}^{n}\right)-\log \left(c_{i, L}^{n}\right)\right) \leq C_{3} .
\end{aligned}
$$

As a consequence, there exists $C_{4}$ depending only on $\Omega, \alpha, \kappa, T, \Psi_{i}, \eta_{i}, \theta_{i}, \zeta$ and $c_{1}^{0}$ such that

$$
\sum_{n=1}^{N} \Delta t \sum_{K \in \mathcal{T}} m_{K}\left(\mu_{1, K}^{n}-\mu_{2, K}^{n}\right)^{2} \leq C_{4}
$$


Proof. Multiplying (2.3) by $\Delta t \eta_{i} \log \left(c_{i, K}^{n}\right)$ and summing over $i \in\{1,2\}, n \in\{1, \ldots, N\}$ and $K \in \mathcal{T}$ yields

$$
A_{1}+A_{2}+A_{3}+A_{4}=0
$$

where we have set

$$
\begin{aligned}
& A_{1}=\sum_{i \in\{1,2\}} \eta_{i} \sum_{n=1}^{N} \sum_{K \in \mathcal{T}} m_{K}\left(c_{i, K}^{n}-c_{i, K}^{n-1}\right) \log \left(c_{i, K}^{n}\right), \\
& A_{2}=\sum_{i \in\{1,2\}} \sum_{n=1}^{N} \Delta t \sum_{\substack{\sigma \in \mathcal{E}_{\text {int }} \\
\sigma=K \mid L}} \tau_{\sigma} c_{i, \sigma}^{n}\left(\mu_{i, K}^{n}-\mu_{i, L}^{n}\right)\left(\log \left(c_{i, K}^{n}\right)-\log \left(c_{i, L}^{n}\right)\right), \\
& A_{3}=\sum_{i \in\{1,2\}} \sum_{n=1}^{N} \Delta t \sum_{\substack{\sigma \in \mathcal{E}_{\text {int }} \\
\sigma=K \mid L}} \tau_{\sigma} c_{i, \sigma}^{n}\left(\Psi_{i, K}-\Psi_{i, L}\right)\left(\log \left(c_{i, K}^{n}\right)-\log \left(c_{i, L}^{n}\right)\right), \\
& A_{4}=\sum_{n=1}^{N} \Delta t \sum_{i \in\{1,2\}} \eta_{i} \theta_{i, \mathcal{T}} \sum_{\substack{\sigma \in \mathcal{E}_{\text {int }} \\
\sigma=K \mid L}} \tau_{\sigma}\left(c_{i, K}^{n}-c_{i, L}^{n}\right)\left(\log \left(c_{i, K}^{n}\right)-\log \left(c_{i, L}^{n}\right)\right) .
\end{aligned}
$$

It follows from the convexity of $H$ that

$$
\begin{aligned}
A_{1} & \geq \sum_{i \in\{1,2\}} \eta_{i} \sum_{n=1}^{N} \sum_{K \in \mathcal{T}} m_{K}\left(H\left(c_{i, K}^{n}\right)-H\left(c_{i, K}^{n-1}\right)\right)=\sum_{i \in\{1,2\}} \eta_{i} \sum_{K \in \mathcal{T}} m_{K}\left(H\left(c_{i, K}^{N}\right)-H\left(c_{i, K}^{0}\right)\right) . \\
& \geq-\sum_{i \in\{1,2\}} \eta_{i} \sum_{K \in \mathcal{T}} m_{K} \geq-C .
\end{aligned}
$$

The particular choice (2.4) for $c_{i, \sigma}^{n}$ was fixed so that

$$
c_{i, \sigma}^{n}\left(\log \left(c_{i, K}^{n}\right)-\log \left(c_{i, L}^{n}\right)\right)=c_{i, K}^{n}-c_{i, L}^{n}, \quad n \geq 1, \sigma=K \mid L .
$$

Therefore, using (2.6) and Cauchy-Schwarz inequality, we deduce that

$$
\begin{aligned}
A_{3} & =\sum_{n=1}^{N} \Delta t \sum_{\substack{\sigma \in \mathcal{E}_{\text {int }} \\
\sigma=K \mid L}} \tau_{\sigma}\left(c_{1, K}^{n}-c_{1, L}^{n}\right)\left(\Psi_{1, K}-\Psi_{1, L}-\Psi_{2, K}+\Psi_{2, L}\right) \\
& \geq-\sum_{n=1}^{N} \Delta t\left(\sum_{\substack{\sigma \in \mathcal{E}_{\text {int }} \\
\sigma=K \mid L}} \tau_{\sigma}\left(c_{1, K}^{n}-c_{1, L}^{n}\right)^{2}\right)^{1 / 2} \sum_{i \in\{1,2\}}\left(\sum_{\substack{\sigma \in \mathcal{E}_{\text {int }} \\
\sigma=K \mid L}} \tau_{\sigma}\left(\Psi_{i, K}-\Psi_{i, L}\right)^{2}\right)^{1 / 2} \geq-C,
\end{aligned}
$$

where the last inequality is a consequence of Corollary 3.4 and of estimate

$$
\sum_{\substack{\sigma \in \mathcal{E}_{\text {int }} \\ \sigma=K \mid L}} \tau_{\sigma}\left(\Psi_{i, K}-\Psi_{i, L}\right)^{2} \leq C
$$

which itself is a consequence of Lemma 9.4 from [16] and of the $H^{1}(\Omega)$ regularity of the external potentials $\Psi_{i}$. Similarly, one can rewrite

$$
A_{2}=\sum_{n=1}^{N} \Delta t \sum_{\substack{\sigma \in \mathcal{E}_{\text {int }} \\ \sigma=K \mid L}} \tau_{\sigma}\left(c_{1, K}^{n}-c_{1, L}^{n}\right)\left(\mu_{1, K}^{n}-\mu_{2, K}^{n}-\mu_{1, L}^{n}+\mu_{2, L}^{n}\right),
$$




$$
=\sum_{n=1}^{N} \Delta t \sum_{K \in \mathcal{T}} m_{K}\left(\frac{1}{m_{K}} \sum_{\substack{\sigma \in \mathcal{E}_{K, \text { int }} \\ \sigma=K \mid L}} \tau_{\sigma}\left(c_{1, K}^{n}-c_{1, L}^{n}\right)\right)\left(\mu_{1, K}^{n}-\mu_{2, K}^{n}\right) .
$$

Thanks to the relation (2.5), it turns to

$$
\begin{aligned}
A_{2}= & \alpha \sum_{n=1}^{N} \Delta t \sum_{K \in \mathcal{T}} m_{K}\left(\frac{1}{m_{K}} \sum_{\begin{array}{c}
\sigma \in \mathcal{E}_{K, \text { int }} \\
\sigma=K \mid L
\end{array}} \tau_{\sigma}\left(c_{1, K}^{n}-c_{1, L}^{n}\right)\right)^{2} \\
& +\kappa \sum_{n=1}^{N} \Delta t \sum_{K \in \mathcal{T}} m_{K}\left(\frac{1}{m_{K}} \sum_{\substack{\sigma \in \mathcal{E}_{K, \text { int }} \\
\sigma=K \mid L}} \tau_{\sigma}\left(c_{1, K}^{n}-c_{1, L}^{n}\right)\right)\left(1-2 c_{1, K}^{n-1}\right) .
\end{aligned}
$$

Using the fact that $0 \leq c_{1, K}^{n-1} \leq 1$ and the inequality $a b \geq-\frac{\alpha}{2 \kappa} a^{2}-\frac{\kappa}{2 \alpha} b^{2}$, we obtain

$$
A_{2} \geq \frac{\alpha}{2} \sum_{n=1}^{N} \Delta t \sum_{K \in \mathcal{T}} m_{K}\left(\frac{1}{m_{K}} \sum_{\substack{\sigma \in \mathcal{E}_{K, \text { int }} \\ \sigma=K \mid L}} \tau_{\sigma}\left(c_{1, K}^{n}-c_{1, L}^{n}\right)\right)^{2}-C .
$$

The combination of (3.13)-(3.17) in (3.12) provides (3.10). Let us now focus on estimate (3.11). Equality (2.5) gives

$$
\begin{aligned}
\sum_{n=1}^{N} \Delta t \sum_{K \in \mathcal{T}} m_{K}\left(\mu_{1, K}^{n}-\mu_{2, K}^{n}\right)^{2} \leq & 2 \alpha^{2} \sum_{n=1}^{N} \Delta t \sum_{K \in \mathcal{T}} m_{K}\left(\frac{1}{m_{K}} \sum_{\substack{\sigma \in \mathcal{E}_{K, \text { int }} \\
\sigma=K \mid L}} \tau_{\sigma}\left(c_{1, K}^{n}-c_{1, L}^{n}\right)\right)^{2} \\
& +2 \kappa^{2} \sum_{n=1}^{N} \Delta t \sum_{K \in \mathcal{T}} m_{K}\left(1-2 c_{1, K}^{n-1}\right)^{2}
\end{aligned}
$$

Since $0 \leq c_{1, K}^{n-1} \leq 1$ and the logarithmic function is increasing, estimate (3.10) concludes the proof.

The following lemma is a transposition to the discrete setting of the weighted estimate (1.14) on the chemical potentials.

Lemma 3.6. There exists $C_{5}$ depending only on $\alpha, \kappa, c_{i}^{0}, \Psi_{i}, T, \Omega, \eta_{i}$ and $\zeta$ such that

$$
\sum_{n=1}^{N} \Delta t \sum_{i \in\{1,2\}} \sum_{\substack{\sigma \in \mathcal{E}_{\text {int }} \\ \sigma=K \mid L}} \tau_{\sigma} c_{i, \sigma}^{n}\left(\mu_{i, K}^{n}-\mu_{i, L}^{n}\right)^{2} \leq C_{5} .
$$

Proof. Definition (2.9) of $\mathfrak{D}_{\mathcal{T}}\left(\boldsymbol{c}^{n}, \boldsymbol{\mu}^{n}\right)$ together with inequality $(a+b+c)^{2} \leq 3\left(a^{2}+b^{2}+c^{2}\right)$ yield 


$$
\begin{aligned}
\frac{1}{\max _{i} \eta_{i}} \sum_{n=1}^{N} \Delta t \sum_{i \in\{1,2\}} \sum_{\substack{\sigma \in \mathcal{E}_{\text {int }} \\
\sigma=K \mid L}} \tau_{\sigma} c_{i, \sigma}^{n}\left(\mu_{i, K}^{n}-\mu_{i, L}^{n}\right)^{2} \leq 3 \sum_{n=1}^{N} \Delta t \mathfrak{D}_{\mathcal{T}}\left(\boldsymbol{c}^{n}, \boldsymbol{\mu}^{n}\right) \\
+3 \sum_{n=1}^{N} \Delta t \sum_{i \in\{1,2\}} \sum_{\substack{\sigma \in \mathcal{E}_{\text {int }} \\
\sigma=K \mid L}} \tau_{\sigma} \frac{c_{i, \sigma}^{n}}{\eta_{i}}\left(\left(\Psi_{i, K}-\Psi_{i, L}\right)^{2}+\left(\theta_{i, \mathcal{T}} \eta_{i}\right)^{2}\left(\log \left(c_{i, K}^{n}\right)-\log \left(c_{i, L}^{n}\right)\right)^{2}\right) .
\end{aligned}
$$

Owing to Lemma 3.3, the first term of the right-hand side is bounded by

$$
\sum_{n=1}^{N} \Delta t \mathfrak{D}_{\mathcal{T}}\left(\boldsymbol{c}^{n}, \boldsymbol{\mu}^{n}\right) \leq \mathfrak{E}_{\mathcal{T}}\left(\boldsymbol{c}^{0}\right)-\mathfrak{E}_{\mathcal{T}}\left(\boldsymbol{c}^{N}\right) \leq 2 \mathfrak{E}_{\mathcal{T}}\left(c^{0}\right)
$$

which is bounded as already seen in the proof of Corollary 3.4. On the other hand, since $0 \leq c_{i, \sigma}^{n} \leq 1$, one has

$$
\sum_{n=1}^{N} \Delta t \sum_{i \in\{1,2\}} \sum_{\substack{\sigma \in \mathcal{E}_{\text {int }} \\ \sigma=K \mid L}} \tau_{\sigma} \frac{c_{i, \sigma}^{n}}{\eta_{i}}\left(\Psi_{i, K}-\Psi_{i, L}\right)^{2} \leq T C_{2} \sum_{i \in\{1,2\}} \frac{1}{\eta_{i}}\left\|\Psi_{i}\right\|_{H^{1}(\Omega)} .
$$

Finally,

$$
\sum_{n=1}^{N} \Delta t \sum_{i \in\{1,2\}} \eta_{i} \sum_{\substack{\sigma \in \mathcal{E}_{\text {int }} \\ \sigma=K \mid L}} \tau_{\sigma} c_{i, \sigma}^{n}\left(\theta_{i, \mathcal{T}}\right)^{2}\left(\log \left(c_{i, K}^{n}\right)-\log \left(c_{i, L}^{n}\right)\right)^{2} \leq C_{3} \max _{i} \theta_{i, \mathcal{T}}
$$

thanks to Lemma 3.5 .

Relation (2.6) guarantees that the sum of the volume fractions is constant equal to 1 in the cells. But this is no longer true on the edges. As shown in the following lemma, the sum of the edge volume fractions is always lower or equal to 1 . Assume for instance that for some $\sigma=K \mid L, c_{1, K}^{n}=1$ and $c_{1, L}^{n}=0$, then both $c_{1, \sigma}^{n}$ and $c_{2, \sigma}^{n}$ are equal to 0 . This degeneracy may lead to severe difficulties in the effective resolution of the nonlinear system provided by the scheme. Next lemma shows that this situation can not be encountered provided the time step is large enough with respect to the size of the mesh. The estimate we provide is based on the worst case scenario and is thus extremely pessimistic. Practically, the inverse CFL condition (3.18) is not needed as soon as the ratio $\alpha / \kappa$ is large enough with respect to the size of the discretization.

Lemma 3.7. Assume that there exists $\gamma>1$ such that

$$
\frac{\Delta t}{m_{K}} \geq \gamma \frac{C_{3}}{\tau_{\star}^{2}}, \quad \forall K \in \mathcal{T}
$$

then there exists $\delta \in(0,1)$ depending on $\tau_{\star}, \tau^{\star}, \ell^{\star}$ and $\gamma$ such that

$$
\left|c_{i, K}^{n}-c_{i, L}^{n}\right| \leq 1-\delta, \quad \forall \sigma=K \mid L \in \mathcal{E}_{\text {int }}, \forall n \in\{1, \ldots, N\}, \forall i \in\{1,2\} .
$$

As a consequence, there exists $c_{\star}>0$ depending only on $\delta$ such that

$$
1 \geq c_{1, \sigma}^{n}+c_{2, \sigma}^{n} \geq c_{\star}, \quad \forall \sigma=K \mid L \in \mathcal{E}_{\mathrm{int}}, \forall n \in\{1, \ldots, N\} .
$$


Proof. Let us first establish (3.19). As a consequence of Lemma 3.5, there holds

$$
\left(\sum_{\substack{\sigma \in \mathcal{E}_{K, \text { int }}, \sigma=K \mid L}} \tau_{\sigma}\left(c_{i, K}^{n}-c_{i, L}^{n}\right)\right)^{2} \leq \frac{C_{3} m_{K}}{\Delta t}, \quad \forall K \in \mathcal{T}, \forall n \in\{1, \ldots, N\}, \forall i \in\{1,2\} .
$$

Let $\sigma=K \mid L \in \mathcal{E}_{\text {int }}$ such that $c_{i, K}^{n}-c_{i, L}^{n} \geq 1-\delta$, then in particular $c_{i, K}^{n} \geq 1-\delta$, so that $c_{i, K}^{n}-c_{i, M}^{n} \geq-\delta$ for all $M \in \mathcal{E}_{K, \text { int }}$. Plugging it in (3.21) and using (2.14) yields

$$
\left(-\tau^{\star}\left(\# \mathcal{E}_{K}-1\right) \delta+\tau_{\star}(1-\delta)\right)^{2} \leq \frac{C_{3} m_{K}}{\Delta t} .
$$

For $\delta \leq \frac{\tau_{\star}}{\left(\ell^{\star}-1\right) \tau^{\star}+\tau_{\star}}$, this yields

$$
\delta \geq \frac{\tau_{\star}-\sqrt{\frac{C_{3} \max _{K \in \mathcal{T}} m_{K}}{\Delta t}}}{\left(\ell^{\star}-1\right) \tau^{\star}+\tau_{\star}} \geq \frac{\tau_{\star}\left(1-\frac{1}{\sqrt{\gamma}}\right)}{\left(\ell^{\star}-1\right) \tau^{\star}+\tau_{\star}}
$$

thanks to (3.18). Thus (3.19) holds with

$$
\delta=\min \left\{\frac{\tau_{\star}}{\left(\ell^{\star}-1\right) \tau^{\star}+\tau_{\star}}, \frac{\tau_{\star}\left(1-\frac{1}{\sqrt{\gamma}}\right)}{\left(\left(\ell^{\star}-1\right) \tau^{\star}+\tau_{\star}\right)}\right\} \in(0,1) .
$$

Let us now turn to the proof of (3.20). If $c_{i, \sigma}^{n}=c_{i, K}^{n}=c_{i, L}^{n}$, we have immediately $c_{1, \sigma}^{n}+c_{2, \sigma}^{n}=1$. Otherwise, the inequality $c_{1, \sigma}^{n}+c_{2, \sigma}^{n} \leq 1$ follows directly from the fact that the logarithmic mean is smaller than the arithmetic one. Define the continuous function $\varphi:[0,1]^{2} \rightarrow[0,1]$ by

$$
\varphi(a, b)=(a-b)\left[\frac{1}{\log (a / b)}-\frac{1}{\log ((1-a) /(1-b))}\right] \quad \text { if } a \neq b, \quad \text { and } \quad \varphi(a, a)=1,
$$

so that, in view of (2.4) and (2.6), one has

$$
c_{1, \sigma}^{n}+c_{2, \sigma}^{n}=\varphi\left(c_{1, K}^{n}, c_{1, L}^{n}\right), \quad \forall \sigma=K \mid L \in \mathcal{E}_{\text {int }}, \forall n \in\{1, \ldots, N\} .
$$

Note that $\varphi(a, b)=0$ if and only if $\{a, b\}=\{0,1\}$. In particular, $\varphi$ is positive on the compact set

$$
\mathcal{K}_{\delta}=\left\{(a, b) \in[0,1]^{2}|| a-b \mid \leq 1-\delta\right\} .
$$

Thus it remains bounded away from 0 by some $c_{\star}$ depending only on $\delta$. Then (3.20) follows from (3.19) and (3.22).

With Lemma 3.7 at hand, we are in position to prove our next lemma, whose goal is to provide first a $L^{2}((0, T) ; B V(\Omega))$ estimate on the approximate mean chemical potential $\bar{\mu}_{\mathcal{T}, \Delta t}$, and then a non-weighted $L^{2}\left(Q_{T}\right)$ estimates on the chemical potentials.

Lemma 3.8. Provided (3.20) holds, there exists $C_{6}$ and $C_{7}$ depending only on $\alpha, \kappa, c_{i}^{0}, \eta_{i}, \Psi_{i}, \theta_{i}, T, \Omega, \zeta, c^{\star}$ such that

$$
\sum_{n=1}^{N} \Delta t\left(\sum_{\substack{\sigma \in \mathcal{E}_{\text {int }} \\ \sigma=K \mid L}} m_{\sigma}\left|\bar{\mu}_{K}^{n}-\bar{\mu}_{L}^{n}\right|\right)^{2} \leq C_{6}
$$

and

$$
\sum_{n=1}^{N} \Delta t \sum_{K \in \mathcal{T}} m_{K}\left(\mu_{i, K}^{n}\right)^{2} \leq C_{7}, \quad i \in\{1,2\} .
$$


Proof. Let $n \geq 1$ and $\sigma=K \mid L \in \mathcal{E}_{\text {int }}$, then thanks to (3.20), either $c_{1, \sigma}^{n} \geq \frac{c^{\star}}{2}$ or $c_{2, \sigma}^{n} \geq \frac{c^{\star}}{2}$. Let us assume that $c_{1, \sigma}^{n} \geq \frac{c^{\star}}{2}$, the other case being similar. We can also assume without loss of generality that $c_{2, K}^{n} \geq c_{2, \sigma}^{n} \geq c_{2, L}^{n}$. Then the triangle inequality ensures that

$$
\left|\bar{\mu}_{K}^{n}-\bar{\mu}_{L}^{n}\right| \leq \sum_{i \in\{1,2\}} c_{i, L}^{n}\left|\mu_{i, K}^{n}-\mu_{i, L}^{n}\right|+\left|\sum_{i \in\{1,2\}} \mu_{i, K}^{n}\left(c_{i, K}^{n}-c_{i, L}^{n}\right)\right| .
$$

Using relation (2.6), the second term of the right-hand side rewrites

$$
\left|\sum_{i \in\{1,2\}} \mu_{i, K}^{n}\left(c_{i, K}^{n}-c_{i, L}^{n}\right)\right|=\left|c_{1, K}^{n}-c_{1, L}^{n}\right|\left|\mu_{1, K}^{n}-\mu_{2, K}^{n}\right|,
$$

while since $c_{2, \sigma}^{n} \geq c_{2, L}^{n}$ and $c_{1, L}^{n} \leq 1 \leq \frac{2 c_{1, \sigma}^{n}}{c^{\star}}$, the first term can be estimated by

$$
\sum_{i \in\{1,2\}} c_{i, L}^{n}\left|\mu_{i, K}^{n}-\mu_{i, L}^{n}\right| \leq \frac{2}{c^{\star}} \sum_{i \in\{1,2\}} c_{i, \sigma}^{n}\left|\mu_{i, K}^{n}-\mu_{i, L}^{n}\right| .
$$

Therefore, using $(a+b+c)^{2} \leq 3\left(a^{2}+b^{2}+c^{2}\right)$, we get that

$$
\left(\sum_{\substack{\sigma \in \mathcal{E}_{\text {int }} \\ \sigma=K \mid L}} m_{\sigma}\left|\bar{\mu}_{K}^{n}-\bar{\mu}_{L}^{n}\right|\right)^{2} \leq A_{n}+B_{n}
$$

where we have set

$$
\begin{aligned}
& A_{n}=\frac{12}{\left(c^{\star}\right)^{2}} \sum_{i \in\{1,2\}}\left(\sum_{\substack{\sigma \in \mathcal{E}_{\text {int }} \\
\sigma=K \mid L}} m_{\sigma} c_{i, \sigma}^{n}\left|\mu_{i, K}^{n}-\mu_{i, L}^{n}\right|\right)^{2}, \\
& B_{n}=3\left(\sum_{\substack{\sigma \in \mathcal{E}_{\text {int }} \\
\sigma=K \mid L}} m_{\sigma}\left|c_{1, K}^{n}-c_{1, L}^{n}\right|\left|\mu_{1, K}^{n}-\mu_{2, K}^{n}\right|\right)^{2} .
\end{aligned}
$$

Using Cauchy-Schwarz inequality, we get that

$$
A_{n} \leq \frac{12}{\left(c^{\star}\right)^{2}} \sum_{i \in\{1,2\}}\left(\sum_{\substack{\sigma \in \mathcal{E}_{\text {int }} \\ \sigma=K \mid L}} \tau_{\sigma} c_{i, \sigma}^{n}\left(\mu_{i, K}^{n}-\mu_{i, L}^{n}\right)^{2}\right)\left(\sum_{\substack{\sigma \in \mathcal{E}_{\text {int }} \\ \sigma=K \mid L}} m_{\sigma} d_{\sigma} c_{i, \sigma}^{n}\right) .
$$

We deduce from $0 \leq c_{i, \sigma}^{n} \leq 1$, from $m_{\sigma} d_{\sigma}=2 m_{D_{\sigma}}$ and from Lemma 3.6 that

$$
\sum_{n=1}^{N} \Delta t A_{n} \leq \frac{24|\Omega|}{\left(c^{\star}\right)^{2}} C_{5} .
$$

Besides, Cauchy-Schwarz inequality yields

$$
B_{n} \leq 3\left(\sum_{\substack{\sigma \in \mathcal{E}_{\text {int }} \\ \sigma=K \mid L}} \tau_{\sigma}\left(c_{1, K}^{n}-c_{1, L}^{n}\right)^{2}\right)\left(\sum_{\substack{\sigma \in \mathcal{E}_{\text {int }} \\ \sigma=K \mid L}} m_{\sigma} d_{\sigma}\left(\left(\mu_{1, K}^{n}-\mu_{2, K}^{n}\right)^{2}+\left(\mu_{1, L}^{n}-\mu_{2, L}^{n}\right)^{2}\right)\right) .
$$


The first term in the right hand side is bounded uniformly w.r.t. $n$ thanks to Corollary 3.4. Reorganizing the second term, one gets that

$$
B_{n} \leq 6 C_{1} \sum_{K \in \mathcal{T}}\left(\sum_{\sigma \in \mathcal{E}_{K, \text { int }}} m_{D_{\sigma}}\right)\left(\mu_{1, K}^{n}-\mu_{2, K}^{n}\right)^{2} .
$$

Thanks to assumption (2.11) on the regularity of the mesh, one has $\sum_{\sigma \in \mathcal{E}_{K, \text { int }}} m_{D_{\sigma}} \leq \zeta m_{K}$. Therefore, it follows from Lemma 3.5 that

$$
\sum_{n=1}^{N} \Delta t B_{n} \leq 6 \zeta C_{1} C_{4}
$$

Combining (3.26) and (3.27) in (3.25) provides (3.23).

The combination of the $L^{2}((0, T) ; B V(\Omega))$ estimate (3.23) with the zero mean condition (2.7) allows to make use of a Poincaré-Sobolev inequality (see for instance [21], [17], Lem. 5.1 or [5]). This provides the following uniform $L^{2}\left(Q_{T}\right)$ estimate on the discrete global chemical potential (recall here that $\Omega \subset \mathbb{R}^{2}$ ):

$$
\sum_{n=1}^{N} \Delta t \sum_{K \in \mathcal{T}} m_{K}\left(\bar{\mu}_{K}^{n}\right)^{2} \leq C .
$$

The definition (2.7) of $\bar{\mu}_{K}^{n}$ and the equation (2.6) provide the following relations:

$$
\mu_{1, K}^{n}=\bar{\mu}_{K}^{n}+c_{2, K}^{n}\left(\mu_{1, K}^{n}-\mu_{2, K}^{n}\right), \quad \mu_{2, K}^{n}=\bar{\mu}_{K}^{n}-c_{1, K}^{n}\left(\mu_{1, K}^{n}-\mu_{2, K}^{n}\right) .
$$

As a result of Lemmas 3.2, 3.5 and Estimate (3.28), we recover (3.24).

\subsection{Existence of a discrete solution}

We are now in position to finish the proof of Theorem 2.3 by showing the existence of (at least) one discrete solution to the scheme $(2.3)-(2.7)$.

Proposition 3.9. There exists at least one solution to the scheme (2.3)-(2.7) satisfying the a priori estimates established in Section 3.1.

Proof. The proof relies on a topological degree argument $[12,24]$. Our goal is to pass continuously from a linear problem for which the existence and uniqueness of the solution is known to the nonlinear system given by our scheme. Since the construction of such an homotopy (which is parametrized by $\lambda \in[0,1]$ ) is non-trivial, we give here a description of it, as well as of the key estimates that allow us to use this machinery.

We assume that $\boldsymbol{c}^{n-1} \in[0,1]^{\# \mathcal{T}}$ is given. For $\lambda \in[0,1]$, we define the nondecreasing functions $f_{\lambda}$ and $p_{\lambda}$ by

$$
f_{\lambda}(c)=\min \left(\frac{1+\lambda}{2}, \max \left(\frac{1-\lambda}{2}, c\right)\right), \quad p_{\lambda}(c)=\int_{1}^{c} \frac{f_{\lambda}^{\prime}(a)}{f_{\lambda}(a)} \mathrm{d} a
$$

so that $f_{\lambda}(c) \geq 0$ and $f_{\lambda}(c)+f_{\lambda}(1-c)=1$ for all $c \in \mathbb{R}$.

We look for the solutions $\left(\boldsymbol{c}^{\lambda}, \boldsymbol{\mu}^{\lambda}\right)=\left(\left(c_{1, K}^{\lambda}, c_{2, K}^{\lambda}\right)_{K \in \mathcal{T}},\left(\mu_{1, K}^{\lambda}, \mu_{2, K}^{\lambda}\right)_{K \in \mathcal{T}}\right)$ of the following modified system. First, equation (2.3) is replaced by

$$
\begin{aligned}
m_{K} \frac{c_{i, K}^{\lambda}-c_{i, K}^{n-1}}{\Delta t} & +\sum_{\substack{\sigma \in \mathcal{E}_{K, \text { int }} \\
\sigma=K \mid L}} \tau_{\sigma} \frac{f_{i, \sigma}^{\lambda}}{\eta_{i}}\left(\mu_{i, K}^{\lambda}-\mu_{i, L}^{\lambda}+\Psi_{i, K}-\Psi_{i, L}\right) \\
& +\theta_{i, \mathcal{T}} \sum_{\substack{\sigma \in \mathcal{E}_{K, \text { int }} \\
\sigma=K \mid L}} \tau_{\sigma}\left(f_{\lambda}\left(c_{i, K}^{\lambda}\right)-f_{\lambda}\left(c_{i, L}^{\lambda}\right)\right)=0, \quad \forall K \in \mathcal{T},
\end{aligned}
$$


where for all $\sigma=K \mid L \in \mathcal{E}_{\text {int }}$ we have set

$$
f_{i, \sigma}^{\lambda}= \begin{cases}\frac{1-\lambda}{2} & \text { if } c_{i, K}^{\lambda} \leq \frac{1-\lambda}{2} \text { and } c_{i, L}^{\lambda} \leq \frac{1-\lambda}{2}, \\ \frac{1+\lambda}{2} & \text { if } c_{i, K}^{\lambda} \geq \frac{1+\lambda}{2} \text { and } c_{i, L}^{\lambda} \geq \frac{1+\lambda}{2}, \\ f_{\lambda}\left(c_{i, K}^{\lambda}\right) & \text { if } c_{i, K}^{\lambda}=c_{i, L}^{\lambda} \in\left(\frac{1-\lambda}{2}, \frac{1+\lambda}{2}\right) \\ \frac{f_{\lambda}\left(c_{i, K}^{\lambda}\right)-f_{\lambda}\left(c_{i, L}^{\lambda}\right)}{p_{\lambda}\left(c_{i, K}^{\lambda}\right)-p_{\lambda}\left(c_{i, L}^{\lambda}\right)} & \text { otherwise. }\end{cases}
$$

Equation (2.5) is replaced for all $K \in \mathcal{T}$ by

$$
\mu_{1, K}^{\lambda}-\mu_{2, K}^{\lambda}=\frac{\alpha}{m_{K}} \sum_{\substack{\sigma \in \mathcal{E}_{K, \text { int }} \\ \sigma=K \mid L}} \tau_{\sigma}\left(f_{\lambda}\left(c_{1, K}^{\lambda}\right)-f_{\lambda}\left(c_{1, L}^{\lambda}\right)\right)+(1-\lambda)\left(c_{1, K}^{\lambda}-\frac{1}{2}\right)+\kappa\left(1-2 c_{1, K}^{n-1}\right) .
$$

We keep the linear relation (2.6), i.e., we impose that

$$
c_{1, K}^{\lambda}+c_{2, K}^{\lambda}=1, \quad \forall K \in \mathcal{T} \text {. }
$$

Finally, equation (2.7) is replaced by

$$
\sum_{K \in \mathcal{T}} m_{K} \bar{\mu}_{K}^{\lambda}=0, \quad \text { with } \quad \bar{\mu}_{K}^{\lambda}=f_{\lambda}\left(c_{1, K}^{\lambda}\right) \mu_{1, K}^{\lambda}+f_{\lambda}\left(c_{2, K}^{\lambda}\right) \mu_{2, K}^{\lambda}
$$

For $\lambda=0$, the system (3.30)-(3.34) reduces to the linear system

$$
\begin{aligned}
m_{K} c_{i, K}^{0}+\frac{\Delta t}{2 \eta_{i}} \sum_{\substack{\sigma \in \mathcal{E}_{K, \text { int }} \\
\sigma=K \mid L}} \tau_{\sigma}\left(\mu_{i, K}^{0}-\mu_{i, L}^{0}\right) & =m_{K} c_{i, K}^{n-1}-\frac{\Delta t}{2 \eta_{i}} \sum_{\substack{\sigma \in \mathcal{E}_{K, \text { int }} \\
\sigma=K \mid L}} \tau_{\sigma}\left(\Psi_{i, K}-\Psi_{i, L}\right), & & K \in \mathcal{T}, i \in\{1,2\}, \\
c_{1, K}^{0}+c_{2, K}^{0} & =1, & & K \in \mathcal{T}, \\
\mu_{1, K}^{0}-\mu_{2, K}^{0}-c_{1, K}^{0} & =\kappa\left(1-2 c_{1, K}^{n-1}\right)-\frac{1}{2}, & & K \in \mathcal{T},
\end{aligned}
$$

complemented with the condition

$$
\sum_{K \in \mathcal{T}} m_{K}\left(\mu_{1, K}^{0}+\mu_{2, K}^{0}\right)=0
$$

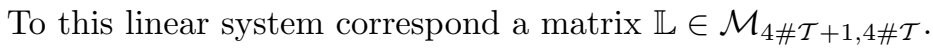

To show that the system (3.35) admits one and only one solution, one first sets the right-hand side to 0 , then multiply (3.35a) by $\mu_{i, K}^{0}$ and sum over $K \in \mathcal{T}$ and $i \in\{1,2\}$. Then using (3.35b) and (3.35c), this provides

$$
\sum_{K \in \mathcal{T}} m_{K}\left|c_{1, K}^{0}\right|^{2}+\sum_{i \in\{1,2\}} \frac{\Delta t}{2 \eta_{i}} \sum_{\substack{\sigma \in \mathcal{E}_{\text {int }} \\ \sigma=K \mid L}} \tau_{\sigma}\left|\mu_{i, K}^{0}-\mu_{i, L}^{0}\right|^{2}=0
$$

from which we infer that $c_{1, K}^{0}=0$ - and thus $c_{2, K}^{0}=0$ because of (3.35b), the right-hand side having been set to 0 - for all $K \in \mathcal{T}$ and that $\mu_{i, K}^{0}$ does not depend on $K$. Moreover, we deduce from (3.35c) with zero right-hand side that $\mu_{1, K}^{0}=\mu_{2, K}^{0}$ for all $K \in \mathcal{T}$. Finally, (3.35d) shows that the discrete chemical potential $\mu_{i, K}^{0}$ are all equal to 0 , hence $\operatorname{Ker}(\mathbb{L})$ is trivial. Therefore, $\mathbb{L}$ has maximal rank and its span can be characterized as the orthogonal 
of one non-zero vector generating $\operatorname{Ker}\left(\mathbb{L}^{T}\right)$. Denote by $\mathbf{1}_{\mathcal{T}}=(1, \ldots, 1)^{T} \in \mathbb{R}^{\# \mathcal{T}}, \mathbf{0}_{\mathcal{T}}=(0, \ldots, 0)^{T} \in \mathbb{R}^{\# \mathcal{T}}$, and by $\boldsymbol{m}_{\mathcal{T}}=\left(m_{K}\right)_{K \in \mathcal{T}} \in \mathbb{R}^{\# \mathcal{T}}$, then one readily checks that

$$
\operatorname{Im}(\mathbb{L})=\left(\begin{array}{c}
\mathbf{1}_{\mathcal{T}} \\
\mathbf{1}_{\mathcal{T}} \\
-\boldsymbol{m}_{\mathcal{T}} \\
\mathbf{0}_{\mathcal{T}} \\
0
\end{array}\right)^{\perp}
$$

since $\mathbf{1}_{\mathcal{T}}$ belongs to the kernel of the discrete Laplacian operator with no-flux boundary conditions. The righthand side of (3.35) then belongs to $\operatorname{Im}(\mathbb{L})$ since $\sum_{K \in \mathcal{T}} \sum_{\substack{\sigma \in \mathcal{E}_{K, \text { int }} \\ \sigma=K \mid L}} \tau_{\sigma}\left(\Psi_{i, K}-\Psi_{i, L}\right)=0$ and $c_{1, K}^{n-1}+c_{2, K}^{n-1}=1$. The linear system (3.35) is then well-posed, hence a topological degree equal to 1 for any large enough set containing its solution.

Let us now establish bounds on the solution to the system (3.30)-(3.34) that are uniform w.r.t. $\lambda \in[0,1]$.

Multiplying (3.30) by $\mu_{i, K}^{\lambda}+\Psi_{i, K}+\eta_{i} \theta_{i, \mathcal{T}} p_{\lambda}\left(c_{i, K}^{\lambda}\right)$ and summing over $K \in \mathcal{T}$ and $i \in\{1,2\}$ provides thanks to the same calculations as in the proof of Lemma 3.3 that

$$
T_{1}^{\lambda}+T_{2}^{\lambda}+T_{3}^{\lambda}+T_{4}^{\lambda}+T_{5}^{\lambda}+\Delta t \mathfrak{D}_{\mathcal{T}}^{\lambda}\left(\boldsymbol{c}^{\lambda}, \boldsymbol{\mu}^{\lambda}\right)=0,
$$

where we have set

$$
\begin{aligned}
T_{1}^{\lambda} & =\alpha \sum_{K \in \mathcal{T}}\left(c_{1, K}^{\lambda}-c_{1, K}^{n-1}\right) \sum_{\substack{\sigma \in \mathcal{E}_{K, \text { int }} \\
\sigma=K \mid L}} \tau_{\sigma}\left(f_{\lambda}\left(c_{1, K}^{n}\right)-f_{\lambda}\left(c_{1, L}^{n}\right)\right), \\
T_{2}^{\lambda} & =(1-\lambda) \sum_{K \in \mathcal{T}} m_{K}\left(c_{1, K}^{\lambda}-c_{1, K}^{n-1}\right)\left(c_{1, K}^{\lambda}-\frac{1}{2}\right), \\
T_{3}^{\lambda} & =\kappa \sum_{K \in \mathcal{T}} m_{K}\left(c_{1, K}^{\lambda}-c_{1, K}^{n-1}\right)\left(1-2 c_{1, K}^{n-1}\right) \\
T_{4}^{\lambda} & =\sum_{i \in\{1,2\}} \sum_{K \in \mathcal{T}} m_{K}\left(c_{i, K}^{\lambda}-c_{i, K}^{n-1}\right) \Psi_{i, K}, \\
T_{5}^{\lambda} & =\sum_{i \in\{1,2\}} \theta_{i, \mathcal{T}} \eta_{i} \sum_{K \in \mathcal{T}} m_{K}\left(c_{i, K}^{\lambda}-c_{i, K}^{n-1}\right) p_{\lambda}\left(c_{i, K}^{\lambda}\right),
\end{aligned}
$$

and

$$
\mathfrak{D}_{\mathcal{T}}^{\lambda}\left(\boldsymbol{c}^{\lambda}, \boldsymbol{\mu}^{\lambda}\right)=\sum_{i \in\{1,2\}} \sum_{\substack{\sigma \in \mathcal{E}_{\text {int }} \\ \sigma=K \mid L}} \tau_{\sigma} \frac{f_{i, \sigma}^{\lambda}}{\eta_{i}}\left|\mu_{i, K}^{\lambda}+\Psi_{i, K}+\eta_{i} \theta_{i, \mathcal{T}} p_{\lambda}\left(c_{i, K}^{\lambda}\right)-\mu_{i, L}^{\lambda}-\Psi_{i, L}-\eta_{i} \theta_{i, \mathcal{T}} p_{\lambda}\left(c_{i, L}^{\lambda}\right)\right|^{2} \geq 0 .
$$

Elementary convexity inequalities yield

$$
T_{2}^{\lambda} \geq \frac{1-\lambda}{2} \sum_{K \in \mathcal{T}} m_{K}\left(\left|c_{1, K}^{\lambda}-\frac{1}{2}\right|^{2}-\left|c_{1, K}^{n-1}-\frac{1}{2}\right|^{2}\right)
$$

and, setting $H_{\lambda}(c)=\int_{1}^{c} p_{\lambda}(a) \mathrm{d} a \geq 0$,

$$
T_{5}^{\lambda} \geq \sum_{i \in\{1,2\}} \theta_{i, \mathcal{T}} \eta_{i} \sum_{K \in \mathcal{T}} m_{K}\left(H_{\lambda}\left(c_{i, K}^{\lambda}\right)-H_{\lambda}\left(c_{i, K}^{n-1}\right)\right) .
$$


On the other hand, using the boundedness of $c_{i, K}^{n-1}$ between 0 and 1 , one gets that

$$
T_{3}^{\lambda} \geq-\kappa \sum_{K \in \mathcal{T}} m_{K}\left|c_{1, K}^{\lambda}\right|-C
$$

while the boundedness of $\Psi_{i, K}$ yields

$$
T_{4}^{\lambda} \geq-\left(\left\|\Psi_{1}\right\|_{\infty}+\left\|\Psi_{2}\right\|_{\infty}\right) \sum_{K \in \mathcal{T}} m_{K}\left|c_{1, K}^{\lambda}\right|-C
$$

Therefore, since $H_{\lambda}(c) \leq H(c)$ for $c \in[0,1]$

$$
T_{2}^{\lambda}+T_{3}^{\lambda}+T_{4}^{\lambda}+T_{5}^{\lambda} \geq \sum_{K \in \mathcal{T}} m_{K} g_{\lambda}\left(c_{1, K}^{\lambda}\right)-C,
$$

where $C$ depends only on $\kappa, \Omega,\left\|\Psi_{i}\right\|_{\infty}, \theta_{i}, \eta_{i}, \rho$ and $h_{\mathcal{T}}$ (but not on $\lambda$ ), and where we have set

$$
g_{\lambda}(c)=\theta_{1, \mathcal{T}} \eta_{1} H_{\lambda}(c)+\theta_{2, \mathcal{T}} \eta_{2} H_{\lambda}(1-c)+\frac{1-\lambda}{2}\left(c-\frac{1}{2}\right)^{2}-\left(\kappa+\left\|\Psi_{1}\right\|_{\infty}+\left\|\Psi_{2}\right\|_{\infty}\right)|c|, \quad c \in \mathbb{R},
$$

with the convention that $g_{\lambda}(c)=+\infty$ if $c \notin[0,1]$ and $\lambda=1$. As a consequence of the technical Lemma A.1 stated in appendix, there exists $C$ depending only $\eta_{i}, \theta_{i}, \rho, h_{\mathcal{T}},\left\|\Psi_{i}\right\|_{\infty}$ and $\kappa$ such that $g_{\lambda}(c) \geq 2\left|c-\frac{1}{2}\right|-C$. Therefore,

$$
T_{2}^{\lambda}+T_{3}^{\lambda}+T_{4}^{\lambda}+T_{5}^{\lambda} \geq \sum_{i \in\{1,2\}} \sum_{K \in \mathcal{T}} m_{K}\left|c_{i, K}^{\lambda}-\frac{1}{2}\right|-C .
$$

Besides, performing a discrete integration by parts on the term $T_{1}^{\lambda}$ yields

$$
T_{1}^{\lambda}=\alpha \sum_{\substack{\sigma \in \mathcal{E}_{\text {int }} \\ \sigma=K \mid L}} \tau_{\sigma}\left[\left(c_{1, K}^{\lambda}-c_{1, L}^{\lambda}\right)-\left(c_{1, K}^{n-1}-c_{1, L}^{n-1}\right)\right]\left(f_{\lambda}\left(c_{1, K}^{\lambda}\right)-f_{\lambda}\left(c_{1, L}^{\lambda}\right)\right)
$$

Since $f_{\lambda}$ is 1-Lipschitz continuous, one has

$$
\begin{aligned}
T_{1}^{\lambda} & \geq \alpha \sum_{\substack{\sigma \in \mathcal{E}_{\text {int }} \\
\sigma=K \mid L}} \tau_{\sigma}\left[\left(f_{\lambda}\left(c_{1, K}^{\lambda}\right)-f_{\lambda}\left(c_{1, L}^{\lambda}\right)\right)^{2}-\left(c_{1, K}^{n-1}-c_{1, L}^{n-1}\right)\left(f_{\lambda}\left(c_{1, K}^{\lambda}\right)-f_{\lambda}\left(c_{1, L}^{\lambda}\right)\right)\right] \\
& \geq \frac{\alpha}{2} \sum_{\substack{\sigma \in \mathcal{E}_{\text {int }} \\
\sigma=K \mid L}} \tau_{\sigma}\left[\left(f_{\lambda}\left(c_{1, K}^{\lambda}\right)-f_{\lambda}\left(c_{1, L}^{\lambda}\right)\right)^{2}-\left(c_{1, K}^{n-1}-c_{1, L}^{n-1}\right)^{2}\right],
\end{aligned}
$$

so that

$$
T_{1}^{\lambda} \geq \frac{\alpha}{2} \sum_{\substack{\sigma \in \mathcal{E}_{\text {int }} \\ \sigma=K \mid L}} \tau_{\sigma}\left(f_{\lambda}\left(c_{1, K}^{\lambda}\right)-f_{\lambda}\left(c_{1, L}^{\lambda}\right)\right)^{2}-C
$$

where $C$ only depends on $\mathcal{T}$ and $\alpha$. Combining (3.38) with (3.37) in (3.36), one gets the existence of $C_{8}$ not depending on $\lambda$ such that, for all $\lambda \in[0,1]$, there holds

$$
\sum_{i \in\{1,2\}} \sum_{K \in \mathcal{T}} m_{K}\left|c_{i, K}^{\lambda}-\frac{1}{2}\right|+\frac{\alpha}{2} \sum_{\substack{\sigma \in \mathcal{E}_{\text {int }} \\ \sigma=K \mid L}} \tau_{\sigma}\left(f_{\lambda}\left(c_{1, K}^{\lambda}\right)-f_{\lambda}\left(c_{1, L}^{\lambda}\right)\right)^{2}+\Delta t \mathfrak{D}_{\mathcal{T}}^{\lambda}\left(\boldsymbol{c}^{\lambda}, \boldsymbol{\mu}^{\lambda}\right) \leq C_{8} .
$$


This implies in particular that $\boldsymbol{c}_{\lambda}$ is bounded independently uniformly w.r.t. $\lambda$, hence

$$
\sum_{K \in \mathcal{T}} m_{K}\left|c_{i, K}^{\lambda}-\frac{1}{2}\right|^{2} \leq C, \quad \forall \lambda \in[0,1], i \in\{1,2\}
$$

for some $C$ not depending on $\lambda$.

We can derive a control on $\boldsymbol{\mu}^{\lambda}$ for $\lambda<1$ from the control of the energy dissipation $\mathfrak{D}_{\mathcal{T}}^{\lambda}$ in (3.39), but this control degenerates as $\lambda$ tends to 1 . To bypass this difficulty, we multiply (3.30) by $\eta_{i} p_{\lambda}\left(c_{i, K}^{\lambda}\right)$. Since $f_{i, \sigma}^{\lambda}$ has been designed so that

$$
f_{i, \sigma}^{\lambda}\left(p_{\lambda}\left(c_{i, K}^{\lambda}\right)-p_{\lambda}\left(c_{i, L}^{\lambda}\right)\right)=f_{\lambda}\left(c_{i, K}^{\lambda}\right)-f_{\lambda}\left(c_{i, L}^{\lambda}\right), \quad \forall \sigma=K \mid L \in \mathcal{E}_{\text {int }},
$$

we can mimic the proof of Lemma 3.5 in order to get the existence of $C$ not depending on $\lambda$ such that

$$
\sum_{K \in \mathcal{T}} m_{K}\left(\frac{1}{m_{K}} \sum_{\substack{\sigma \in \mathcal{E}_{K, \text { int }} \\ \sigma=K \mid L}} \tau_{\sigma}\left(f_{\lambda}\left(c_{1, K}^{\lambda}\right)-f_{\lambda}\left(c_{1, L}^{\lambda}\right)\right)\right)^{2} \leq C,
$$

together with

$$
\sum_{K \in \mathcal{T}} m_{K}\left(\mu_{1, K}^{\lambda}-\mu_{2, K}^{\lambda}\right)^{2} \leq C
$$

Thanks to (3.40), we can reproduce the proof of Lemma 3.7 to claim that

$$
f_{1, \sigma}^{\lambda}+f_{2, \sigma}^{\lambda} \geq f^{\star}, \quad \forall \sigma \in \mathcal{E}_{\text {int }},
$$

for some $f^{\star}>0$ not depending on $\lambda$. This provides a uniform in $\lambda$ discrete $B V$ estimate on $\left(\bar{\mu}_{K}^{\lambda}\right)_{K \in \mathcal{T}}$ and finally the existence of some $C_{9}$ not depending on $\lambda$ following the path of Lemma 3.8 such that

$$
\sum_{i \in\{1,2\}} \sum_{K \in \mathcal{T}} m_{K}\left(\mu_{i, K}^{\lambda}\right)^{2} \leq C_{9}
$$

Then the topological degree corresponding to system (3.30)-(3.34) on the compact set

$$
\mathcal{K}=\left\{\left(\left(c_{1, K}, c_{2, K}\right)_{K \in \mathcal{T}},\left(\mu_{1, K}, \mu_{2, K}\right)_{K \in \mathcal{T}}\right) \mid \sum_{i \in\{1,2\}} \sum_{K \in \mathcal{T}} m_{K}\left(\left|c_{i, K}-\frac{1}{2}\right|+\left(\mu_{i, K}\right)^{2}\right) \leq C_{8}+C_{9}+1\right\}
$$

is constant equal to 1 whatever $\lambda \in[0,1]$. In particular, there exists a solution to our scheme (2.3)-(2.7) which corresponds to the case $\lambda=1$.

The existence of a solution $\left(\boldsymbol{c}^{n}, \boldsymbol{\mu}^{n}\right)$ to the scheme (2.3)-(2.7) for all $n \in\{1, \ldots, N\}$ allows to define a piecewise constant approximate solution $\left(\boldsymbol{c}_{\mathcal{T}, \Delta t}, \boldsymbol{\mu}_{\mathcal{T}, \Delta t}\right)$ by $(2.10)$.

\section{Convergence of the scheme}

The goal of this section is to prove Theorem 2.4, i.e., that $\left(\boldsymbol{c}_{\mathcal{T}, \Delta t}, \mu_{\mathcal{T}, \Delta t}\right)$ tends to a weak solution $(\boldsymbol{c}, \boldsymbol{\mu})$ of (1.1)-(1.6) in a suitable topology as $h_{\mathcal{T}}$ and $\Delta t$ tend to 0 provided the mesh remains sufficiently regular. Consider a sequence of regular meshes $\left(\mathcal{T}_{m}, \mathcal{E}_{m},\left(\boldsymbol{x}_{K}\right)_{K \in \mathcal{T}_{m}}\right)_{m>1}$ such that (2.11)-(2.14) hold for some uniform $\zeta$, $\ell^{\star}, \tau^{\star}$ and $\tau_{\star}$ w.r.t. $m$, and such that $h_{\mathcal{T}_{m}}$ tends to 0 as $m$ tends to $+\infty$, and a sequence of times steps $\left(\Delta t_{m}\right)_{m \geq 1}$ with $\Delta t_{m}=T / N_{m}$ with $N_{m}$ tending to $+\infty$ with $m$. Then the a priori estimates derived in Section 3.1 are satisfied uniformly provided (3.20) holds, as it is the case if the inverse CFL condition (3.18) is fulfilled.

The first lemma gathers some first consequences of the a priori estimates stated in Section 3.1. 
Lemma 4.1. There exist $c_{i} \in L^{\infty}\left(Q_{T} ;[0,1]\right)$ with $c_{1}+c_{2}=1$ and $\mu_{i} \in L^{2}\left(Q_{T}\right), i \in\{1,2\}$ such that, up to a subsequence,

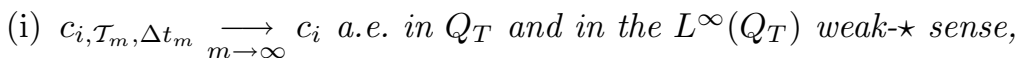

(ii) $\mu_{i, \mathcal{T}_{m}, \Delta t_{m}} \underset{m \rightarrow \infty}{\longrightarrow} \mu_{i}$ weakly in $L^{2}\left(Q_{T}\right)$.

Moreover, $\int_{\Omega} \bar{\mu}(t, \boldsymbol{x}) \mathrm{d} \boldsymbol{x}=0$ for a.e. $t \geq 0$, where $\bar{\mu}=c_{1} \mu_{1}+c_{2} \mu_{2}$.

Proof. Because of Lemma 3.2, the approximate solutions $c_{i, \mathcal{T}_{m}, \Delta t_{m}}$ remain bounded a.e. in $Q_{T}$ between 0 and 1 . Therefore, there exists $c_{i} \in L^{\infty}\left(Q_{T} ;[0,1]\right)$ such that, up to a subsequence, $c_{i, \mathcal{T}_{m}, \Delta t_{m}}$ tends to $c_{i}$ in the $L^{\infty}\left(Q_{T}\right)$ weak- $\star$ sense. This is enough to pass in the limit in the relation $c_{1, \mathcal{T}_{m}, \Delta t_{m}}+c_{2}, \mathcal{T}_{m}, \Delta t_{m}=1$ which directly follows from (2.6). On the other hand, it follows from Lemma 3.8 that the sequences $\left(\mu_{i, \mathcal{T}_{m}, \Delta t_{m}}\right)_{m \geq 1}$ are uniformly bounded in $L^{2}\left(Q_{T}\right)$, hence the weak convergence in $L^{2}\left(Q_{T}\right)$ towards some $\mu_{i}$. The almost everywhere convergence of $c_{i, \mathcal{T}_{m}, \Delta t_{m}}$ towards $c_{i}$ follows from the discrete Aubin-Lions lemma stated in Appendix B. Finally, given an arbitrary $\varphi \in L^{\infty}(0, T)$, then multiplying (2.7) by $\frac{1}{\Delta t} \int_{(n-1) \Delta t_{m}}^{n \Delta t_{m}} \varphi(t) \mathrm{d} t$ and summing over $n \in\left\{1, \ldots, N_{m}\right\}$ yields

$$
\iint_{Q_{T}}\left[c_{1, \mathcal{T}_{m}, \Delta t_{m}} \mu_{1, \mathcal{T}_{m}, \Delta t_{m}}+c_{2, \mathcal{T}_{m}, \Delta t_{m}} \mu_{2, \mathcal{I}_{m}, \Delta t_{m}}\right] \varphi \mathrm{d} \boldsymbol{x} \mathrm{d} t=0 .
$$

Since $c_{i, \mathcal{T}_{m}, \Delta t_{m}}$ converges a.e. towards $c_{i}$ while remaining uniformly bounded between 0 and 1 , it converges also in the strong $L^{2}\left(Q_{T}\right)$ sense thanks to Lebesgue dominated convergence theorem. Together with the weak convergence in $L^{2}\left(Q_{T}\right)$ of $\mu_{i, \mathcal{T}_{m}, \Delta t_{m}}$ towards $\mu_{i}$, we have enough compactness to pass to the limit $m \rightarrow+\infty$ in the above expression, which gives that

$$
\iint_{Q_{T}} \bar{\mu}(t, \boldsymbol{x}) \varphi(t) \mathrm{d} \boldsymbol{x} \mathrm{d} t=0, \quad \forall \varphi \in L^{\infty}\left(Q_{T}\right) .
$$

This of course implies that $\int_{\Omega} \bar{\mu}(t, \boldsymbol{x}) \mathrm{d} \boldsymbol{x}=0$ for a.e. $t \geq 0$.

Before going further, we need to introduce some additional material concerning the construction of a strongly consistent approximate gradient based on the SUSHI finite volume scheme [17]. We gather in the following proposition the properties of this approximate gradient to be used in what follows. The super-admissibility of the mesh is crucial at this point. We refer to [17] or to Chapter 13 of [13] for the proofs corresponding to Proposition 4.2.

Proposition $4.2([17])$. Define by $\mathcal{X}_{\mathcal{T}_{m}, \Delta t_{m}}$ the space of bounded piecewise constant functions per control volume and per time step as $c_{i, \mathcal{T}_{m}, \Delta t_{m}}$ and $\mu_{i, \mathcal{T}_{m}, \Delta t_{m}}$, i.e.,

$$
\mathcal{X}_{\mathcal{T}_{m}, \Delta t_{m}}=\left\{u_{\mathcal{T}_{m}, \Delta t_{m}} \in L^{\infty}\left(Q_{T}\right) \mid u_{\mathcal{T}_{m}, \Delta t_{m}}(t, \boldsymbol{x})=u_{K}^{n} \in \mathbb{R}, \forall(t, \boldsymbol{x}) \in\left(t^{n-1}, t^{n}\right] \times K\right\} .
$$

Then there exists a linear operator $\nabla_{\mathcal{T}_{m}}: \mathcal{X}_{\mathcal{T}_{m}} \rightarrow L^{\infty}\left(Q_{T}\right)^{2}$ such that:

(i) for all $u_{\mathcal{T}_{m}, \Delta t_{m}}, v_{\mathcal{T}_{m}, \Delta t_{m}} \in \mathcal{X}_{\mathcal{T}_{m}, \Delta t_{m}}$ and all $n \in\left\{1, \ldots, N_{m}\right\}$, one has

$$
\int_{\Omega} \nabla_{\mathcal{T}_{m}} u_{\mathcal{T}_{m}, \Delta t_{m}}\left(t^{n}, \boldsymbol{x}\right) \cdot \nabla_{\mathcal{T}_{m}} v_{\mathcal{T}_{m}, \Delta t_{m}}\left(t^{n}, \boldsymbol{x}\right) \mathrm{d} \boldsymbol{x}=\sum_{\substack{\sigma \in \mathcal{E}_{\text {int }, m} \\ \sigma=K \mid L}} \tau_{\sigma}\left(u_{K}^{n}-u_{L}^{n}\right)\left(v_{K}^{n}-v_{L}^{n}\right) ;
$$

(ii) if the sequence $\left(u_{\mathcal{T}_{m}, \Delta t_{m}}\right)_{m>1}$ is such that $\left\|u_{\mathcal{T}_{m}, \Delta t_{m}}\right\|_{L^{2}\left(Q_{T}\right)}$ and $\left\|\nabla_{\mathcal{T}_{m}} u_{\mathcal{T}_{m}, \Delta t_{m}}\right\|_{L^{2}\left(Q_{T}\right)^{2}}$ are bounded w.r.t. $m$, then there exists $u \in L^{2}\left((0, T) ; H^{1}(\Omega)\right)$ such that $u_{\mathcal{T}_{m}, \Delta t_{m}}$ converges weakly towards $u$ in $L^{2}\left(Q_{T}\right)$ and $\nabla_{\mathcal{T}_{m}} u_{\mathcal{T}_{m}, \Delta t_{m}}$ converges weakly towards $\boldsymbol{\nabla} u$ in $L^{2}\left(Q_{T}\right)^{2}$;

(iii) let $\varphi \in C^{\infty}\left(\bar{Q}_{T}\right)$, and define $\varphi_{\mathcal{T}_{m}, \Delta t_{m}}$ by fixing $\varphi_{K}^{n}=\varphi\left(t^{n}, \boldsymbol{x}_{K}\right)$, then $\boldsymbol{\nabla}_{\mathcal{T}_{m}} \varphi_{\mathcal{T}_{m}, \Delta t_{m}}$ converges towards $\boldsymbol{\nabla} \varphi$ in $L^{p}\left(Q_{T}\right)^{2}$ for all $p \in[1, \infty)$; 
(iv) for all $K \in \mathcal{T}_{m}$ and all $n \in\left\{1, \ldots, N_{m}\right\}$, there holds

$$
\int_{K} \boldsymbol{\nabla}_{\mathcal{T}_{m}} u_{\mathcal{T}_{m}, \Delta t_{m}}\left(t^{n}, \boldsymbol{x}\right) \mathrm{d} \boldsymbol{x}=\sum_{\substack{\sigma \in \mathcal{E}_{K, \text { int }, m} \\ \sigma=K \mid L}} d_{K, \sigma} \tau_{\sigma}\left(u_{L}^{n}-u_{K}^{n}\right) \boldsymbol{n}_{K L} .
$$

Let us point out that we could have improved the convergence property in (iii) until obtaining the uniform convergence at the price of adding some additional degrees of freedom on the boundary edges. However, the convergence properties stated in Proposition 4.2 are sufficient to prove the convergence of our scheme. Therefore, we avoid the introduction of additional material.

Next statement is a straightforward consequence of the combination of Proposition 4.2 together with Corollary 3.4.

Corollary 4.3. Up to a subsequence, the approximate gradient $\boldsymbol{\nabla}_{\mathcal{T}_{m}} c_{i, \mathcal{T}_{m}, \Delta t_{m}}$ converges towards $\boldsymbol{\nabla} c_{i}$ in the weak $-\star$ topology of $L^{\infty}\left((0, T) ; L^{2}\left(Q_{T}\right)\right)^{2}$ as $m$ tends to $+\infty$. In particular, $c_{i}$ belongs to $L^{\infty}\left((0, T) ; H^{1}(\Omega)\right)$. Moreover, $\nabla_{\mathcal{T}_{m}} \Psi_{i, \mathcal{T}_{m}}$ converges weakly towards $\nabla \Psi_{i}$.

The purpose of next lemma is twofold. First, one shows that (1.3) and (1.4) are satisfied by the limits $c_{i}, \mu_{i}$. Second, we deduce from this consistency property the approximate gradient of the volume fractions converges strongly in $L^{2}\left(Q_{T}\right)$.

Lemma 4.4. The weak formulation (1.17) holds for all $\varphi \in C_{c}^{\infty}([0, T) \times \bar{\Omega})$. Moreover, $\nabla_{\mathcal{T}_{m}} c_{i, \mathcal{T}_{m}, \Delta t_{m}}$ converges strongly in $L^{2}\left(Q_{T}\right)$ towards $\nabla c_{i}$ as $m$ tends to $+\infty$.

Proof. Let us first establish (1.17). As a preliminary, define the piecewise constant function

$$
\check{c}_{1, \mathcal{T}_{m}, \Delta t_{m}}(t, \boldsymbol{x})=c_{1, K}^{n-1} \quad \text { if }(t, \boldsymbol{x}) \in\left[t^{n-1}, t^{n}\right) \times K, \quad n \in\left\{1, \ldots N_{m}\right\}, K \in \mathcal{T}_{m} .
$$

Then $\check{c}_{1, \mathcal{T}_{m}, \Delta t_{m}}$ remains bounded between 0 and 1 . Therefore,

$$
\iint_{Q_{T}}\left|\check{c}_{1, \mathcal{T}_{m}, \Delta t_{m}}-c_{1, \mathcal{T}_{m}, \Delta t_{m}}\right|^{2} \mathrm{~d} \boldsymbol{x} \mathrm{d} t \leq \Delta t|\Omega|+\iint_{Q_{T-\Delta t_{m}}}\left|c_{1, \mathcal{T}_{m}, \Delta t_{m}}\left(t+\Delta t_{m}, \boldsymbol{x}\right)-c_{1, \mathcal{T}_{m}, \Delta t_{m}}(t, \boldsymbol{x})\right|^{2} \mathrm{~d} \boldsymbol{x} \mathrm{d} t .
$$

Following Lemma 4.1, $\left(c_{1, \mathcal{T}_{m}, \Delta t_{m}}\right)_{m>1}$ converges in $L^{2}\left(Q_{T}\right)$. The reciprocal of the Riesz-Fréchet-Kolmogorov theorems allows us to claim that the second term in the right-hand side tends to 0 as $m$ tends to $+\infty$. Therefore, $\check{c}_{1, \mathcal{T}_{m}, \Delta t_{m}}$ tends to $c_{1}$ strongly in $L^{2}\left(Q_{T}\right)$ together with $c_{1, \mathcal{T}_{m}, \Delta t_{m}}$.

Given an arbitrary $\varphi \in C^{\infty}\left(\bar{Q}_{T}\right)$, we define $\varphi_{K}^{n}=\varphi\left(t^{n}, \boldsymbol{x}_{K}\right)$ for all $n \in\left\{1, \ldots, N_{m}\right\}$ and all $K \in \mathcal{T}_{m}$. Multiplying (2.5) by $\varphi_{K}^{n}$ and summing over $n$ and $K$ yields

$$
\begin{aligned}
& \iint_{Q_{T}}\left(\mu_{1, \mathcal{T}_{m}, \Delta t_{m}}\right.\left.-\mu_{2, \mathcal{T}_{m}, \Delta t_{m}}\right) \varphi_{\mathcal{T}_{m}, \Delta t_{m}} \mathrm{~d} \boldsymbol{x} \mathrm{d} t \\
&=\alpha \iint_{Q_{T}} \nabla_{\mathcal{T}_{m}} c_{1, \mathcal{T}_{m}, \Delta t_{m}} \cdot \nabla_{\mathcal{T}_{m}} \varphi_{\mathcal{T}_{m}, \Delta t_{m}} \mathrm{~d} \boldsymbol{x} \mathrm{d} t+\kappa \iint_{Q_{T}}\left(1-2 \check{c}_{1, \mathcal{T}_{m}, \Delta t_{m}}\right) \varphi_{\mathcal{T}_{m}, \Delta t_{m}} \mathrm{~d} \boldsymbol{x} \mathrm{d} t .
\end{aligned}
$$

We can pass to the limit $m \rightarrow+\infty$ in the previous equality. Since $\mu_{i, \mathcal{T}_{m}, \Delta t_{m}}$ converges weakly towards $\mu_{i}$ in $L^{2}\left(Q_{T}\right)$ thanks to Lemma 4.1 , since $\boldsymbol{\nabla}_{\mathcal{T}_{m}} c_{1, \mathcal{T}_{m}, \Delta t_{m}}$ converges weakly in $L^{2}\left(Q_{T}\right)^{2}$ towards $\nabla c_{1}$ thanks to Corollary 4.3, since $\check{c}_{1, \mathcal{T}_{m}, \Delta t_{m}}$ converges in $L^{2}\left(Q_{T}\right)$ towards $c_{1}$, since $\varphi_{\mathcal{T}_{m}, \Delta t_{m}}$ converges uniformly towards $\varphi$, and since $\boldsymbol{\nabla}_{\mathcal{T}_{m}} \varphi_{\mathcal{T}_{m}, \Delta t_{m}}$ converges towards $\boldsymbol{\nabla} \varphi$ in $L^{2}\left(Q_{T}\right)^{2}$ thanks to Proposition 4.2, one recovers (1.17).

Thanks to a standard density arguments, one checks that (1.17) holds for $\varphi \in L^{2}\left((0, T) ; H^{1}(\Omega)\right)$, thus in particular for $\varphi=c_{1}$, which yields

$$
\alpha \iint_{Q_{T}}\left|\nabla c_{1}\right|^{2} \mathrm{~d} \boldsymbol{x} \mathrm{d} t=\iint_{Q_{T}}\left[\mu_{1}-\mu_{2}-\kappa\left(1-2 c_{1}\right)\right] c_{1} \mathrm{~d} \boldsymbol{x} \mathrm{d} t .
$$


Choosing $\varphi_{\mathcal{T}_{m}, \Delta t_{m}}=c_{1, \mathcal{T}_{m}, \Delta t_{m}}$ in (4.2) and passing to the limit $m \rightarrow+\infty$ shows that

$$
\lim _{m \rightarrow \infty} \iint_{Q_{T}}\left|\nabla_{\mathcal{T}_{m}} c_{1, \mathcal{T}_{m}, \Delta t_{m}}\right|^{2} \mathrm{~d} \boldsymbol{x} \mathrm{d} t=\frac{1}{\alpha} \iint_{Q_{T}}\left[\mu_{1}-\mu_{2}-\kappa\left(1-2 c_{1}\right)\right] c_{1} \mathrm{~d} \boldsymbol{x} \mathrm{d} t=\iint_{Q_{T}}\left|\nabla c_{1}\right|^{2} \mathrm{~d} \boldsymbol{x} \mathrm{d} t
$$

hence the strong convergence of $\nabla_{\mathcal{T}_{m}} c_{1, \mathcal{T}_{m}, \Delta t_{m}}$ towards $\boldsymbol{\nabla} c_{1}$.

Next lemma focuses on the term corresponding to $c_{i} \nabla \mu_{i}$. For $m \geq 1$, we define

$$
\boldsymbol{V}_{i, \sigma}^{n}=2 c_{i, \sigma}^{n} \frac{\mu_{i, K}^{n}-\mu_{i, L}^{n}}{d_{\sigma}} \boldsymbol{n}_{K L}, \quad \forall \sigma=K \mid L \in \mathcal{E}_{\text {int }, m}, \forall n \in\left\{1, \ldots, N_{m}\right\},
$$

and the corresponding piecewise constant vector field

$$
\boldsymbol{V}_{i, \mathcal{D}_{m}, \Delta t_{m}}(t, \boldsymbol{x})= \begin{cases}\boldsymbol{V}_{i, \sigma}^{n} & \text { if }(t, \boldsymbol{x}) \in\left(t^{n-1}, t^{n}\right] \times D_{\sigma}, \sigma \in \mathcal{E}_{\text {int }, m}, \\ 0 & \text { if }(t, \boldsymbol{x}) \in\left(t^{n-1}, t^{n}\right] \times\left(K \backslash \bigcup_{\sigma \in \mathcal{E}_{K, \text { int }}} D_{K, \sigma}\right) .\end{cases}
$$

Lemma 4.5. Let $\boldsymbol{V}_{i, \mathcal{D}_{m}, \Delta t_{m}}$ be defined by (4.3) and (4.4), then, up to a subsequence, $\boldsymbol{V}_{i, \mathcal{D}_{m}, \Delta t_{m}}$ converges weakly towards $-c_{i} \nabla \mu_{i}$ in $L^{2}\left(Q_{T}\right)$ as $m$ tends to $+\infty$.

Proof. Since $m_{\sigma} d_{\sigma}=2 m_{D_{\sigma}}$ and since $0 \leq c_{i, \sigma}^{n} \leq 1$, it results from Lemma 3.6 that

$$
\left\|\boldsymbol{V}_{i, \mathcal{D}_{m}, \Delta t_{m}}\right\|_{L^{2}\left(Q_{T}\right)^{2}}^{2}=2 \sum_{n=1}^{N_{m}} \Delta t \sum_{\substack{\sigma \in \mathcal{E}_{\text {int }, m} \\ \sigma=K \mid L}} \tau_{\sigma}\left(c_{i, \sigma}^{n}\right)^{2}\left(\mu_{i, K}^{n}-\mu_{i, L}^{n}\right)^{2} \leq C .
$$

Therefore, up to a subsequence, $\boldsymbol{V}_{i, \mathcal{D}_{m}, \Delta t_{m}}$ converges weakly in $L^{2}\left(Q_{T}\right)^{2}$ towards some $\boldsymbol{V}_{i}$. Let us identify $\boldsymbol{V}_{i}$ as $-c_{i} \boldsymbol{\nabla} \mu_{i}$. To this end, we introduce an arbitrary smooth vector field $\boldsymbol{\Phi} \in C_{c}^{\infty}\left(Q_{T}\right)^{2}$, and, for all $m \geq 1$, we denote by

$$
\boldsymbol{\Phi}_{K}^{n}=\boldsymbol{\Phi}\left(t^{n}, \boldsymbol{x}_{K}\right), \quad \boldsymbol{\Phi}_{\sigma}^{n}=\frac{1}{m_{\sigma}} \int_{\sigma} \boldsymbol{\Phi}\left(t^{n}, \boldsymbol{x}\right) \mathrm{d} \boldsymbol{x}, \quad \forall K \in \mathcal{T}_{m}, \forall \sigma \in \mathcal{E}_{\mathrm{int}, m}, \forall n \in\left\{1, \ldots, N_{m}\right\},
$$

and by

$$
\begin{aligned}
& \boldsymbol{\Phi}_{\mathcal{T}_{m}, \Delta t_{m}}(t, \boldsymbol{x})=\boldsymbol{\Phi}_{K}^{n} \quad \text { if }(t, \boldsymbol{x}) \in\left(t^{n-1}, t^{n}\right] \times K, \\
& \boldsymbol{\Phi}_{\mathcal{D}_{m}, \Delta t_{m}}(t, \boldsymbol{x})= \begin{cases}\boldsymbol{\Phi}_{\sigma}^{n} & \text { if }(t, \boldsymbol{x}) \in\left(t^{n-1}, t^{n}\right] \times D_{\sigma}, \sigma \in \mathcal{E}_{\mathrm{int}, m}, \\
0 & \text { if }(t, \boldsymbol{x}) \in\left(t^{n-1}, t^{n}\right] \times\left(K \backslash \bigcup_{\sigma \in \mathcal{E}_{K, \text { int }}} D_{K, \sigma}\right),\end{cases}
\end{aligned}
$$

for almost all $(t, \boldsymbol{x}) \in Q_{T}$. Thanks to the regularity of $\boldsymbol{\Phi}$, it is easy to check that both $\boldsymbol{\Phi}_{\mathcal{T}_{m}, \Delta t_{m}}$ and $\boldsymbol{\Phi}_{\mathcal{D}_{m}, \Delta t_{m}}$ converge uniformly towards $\boldsymbol{\Phi}$ as $m$ tends to $+\infty$. This implies in particular that

$$
B_{i, m}(\boldsymbol{\Phi})=\iint_{Q_{T}} \boldsymbol{V}_{i, \mathcal{D}_{m}, \Delta t_{m}}(t, \boldsymbol{x}) \cdot \boldsymbol{\Phi}_{\mathcal{D}_{m}, \Delta t_{m}} \mathrm{~d} \boldsymbol{x} \mathrm{d} t \underset{m \rightarrow \infty}{\longrightarrow} \iint_{Q_{T}} \boldsymbol{V}_{i} \cdot \boldsymbol{\Phi} \mathrm{d} \boldsymbol{x} \mathrm{d} t .
$$

On the other hand, $B_{i, m}(\mathbf{\Phi})$ can be decomposed into

$$
B_{i, m}(\boldsymbol{\Phi})=B_{i, m}^{(1)}(\boldsymbol{\Phi})+B_{i, m}^{(2)}(\boldsymbol{\Phi})+B_{i, m}^{(3)}(\boldsymbol{\Phi})+B_{i, m}^{(4)}(\boldsymbol{\Phi}),
$$

where, denoting by

$$
\bar{c}_{i, \sigma}^{n}=\frac{d_{K, \sigma} c_{i, L}^{n}+d_{L, \sigma} c_{i, K}^{n}}{d_{\sigma}}, \quad \forall \sigma=K \mid L \in \mathcal{E}_{\mathrm{int}, m}, \forall n \in\left\{1, \ldots, N_{m}\right\},
$$


we have set

$$
\begin{aligned}
B_{i, m}^{(1)}(\boldsymbol{\Phi}) & =\sum_{n=1}^{N_{m}} \Delta t_{m} \sum_{\substack{\sigma \in \mathcal{E}_{\text {int }, m} \\
\sigma=K \mid L}} m_{\sigma}\left(c_{i, \sigma}^{n}-\bar{c}_{i, \sigma}^{n}\right)\left(\mu_{i, K}^{n}-\mu_{i, L}^{n}\right) \boldsymbol{\Phi}_{\sigma}^{n} \cdot \boldsymbol{n}_{K \sigma}, \\
B_{i, m}^{(2)}(\boldsymbol{\Phi}) & =\sum_{n=1}^{N_{m}} \Delta t_{m} \sum_{K \in \mathcal{T}_{m}} \mu_{i, K}^{n} c_{i, K}^{n} \sum_{\sigma \in \mathcal{E}_{K, \text { int }}} m_{\sigma} \boldsymbol{\Phi}_{\sigma}^{n} \cdot \boldsymbol{n}_{K \sigma}, \\
B_{i, m}^{(3)}(\boldsymbol{\Phi}) & =\sum_{n=1}^{N_{m}} \Delta t_{m} \sum_{K \in \mathcal{T}_{m}} m_{K} \mu_{i, K}^{n} \boldsymbol{\Phi}_{K}^{n} \cdot\left[\frac{1}{m_{K}} \sum_{\sigma \in \mathcal{E}_{K, \text { int }}} m_{\sigma}\left(\bar{c}_{i, \sigma}^{n}-c_{i, K}^{n}\right) \boldsymbol{n}_{K \sigma}\right], \\
B_{i, m}^{(4)}(\boldsymbol{\Phi}) & =\sum_{n=1}^{N_{m}} \Delta t_{m} \sum_{K \in \mathcal{T}_{m}} \mu_{i, K}^{n} \sum_{\sigma \in \mathcal{E}_{K, \text { int }}} m_{\sigma}\left(\bar{c}_{i, \sigma}^{n}-c_{i, K}^{n}\right)\left(\boldsymbol{\Phi}_{\sigma}^{n}-\boldsymbol{\Phi}_{K}^{n}\right) \cdot \boldsymbol{n}_{K \sigma} .
\end{aligned}
$$

Let us first focus on $B_{i, m}^{(1)}(\mathbf{\Phi})$, which can be controled as follows thanks to Cauchy-Schwarz inequality and the fact that $d_{\sigma} \leq 2 h_{\mathcal{T}}$ :

$$
\begin{aligned}
\left|B_{i, m}^{(1)}(\mathbf{\Phi})\right|^{2} \leq 4 h_{\mathcal{T}}^{2}\|\boldsymbol{\Phi}\|_{\infty}^{2}\left(\sum_{n=1}^{N_{m}} \Delta t_{m} \sum_{\substack{\sigma \in \mathcal{E}_{\text {int }, m} \\
\sigma=K \mid L}} \tau_{\sigma} \frac{\left(c_{i, \sigma}^{n}-\bar{c}_{i, \sigma}^{n}\right)^{2}}{c_{i, \sigma}^{n}}\right) & \times\left(\sum_{n=1}^{N_{m}} \Delta t_{m} \sum_{\substack{\sigma \in \mathcal{E}_{\text {int }, m} \\
\sigma=K \mid L}} \tau_{\sigma} c_{i, \sigma}^{n}\left(\mu_{i, K}^{n}-\mu_{i, L}^{n}\right)^{2}\right) .
\end{aligned}
$$

The second sum in the right-hand side is uniformly bounded thanks to Lemma 3.6, whereas since $\left|c_{i, \sigma}^{n}-\bar{c}_{i, \sigma}^{n}\right| \leq$ $\left|c_{i, K}^{n}-c_{i, L}^{n}\right|$, Lemma 3.5 and the particular definition (2.4) of $c_{i, \sigma}^{n}$ ensure that

$$
\sum_{n=1}^{N_{m}} \Delta t_{m} \sum_{\substack{\sigma \in \mathcal{E}_{\text {int }, m} \\ \sigma=K \mid L}} \tau_{\sigma} \frac{\left(c_{i, \sigma}^{n}-\bar{c}_{i, \sigma}^{n}\right)^{2}}{c_{i, \sigma}^{n}} \leq \sum_{n=1}^{N_{m}} \Delta t_{m} \sum_{\substack{\sigma \in \mathcal{E}_{\text {int }, m} \\ \sigma=K \mid L}} \tau_{\sigma}\left(c_{i, K}^{n}-c_{i, L}^{n}\right)\left(\log \left(c_{i, K}^{n}\right)-\log \left(c_{i, L}^{n}\right)\right) \leq \frac{C_{3}}{\eta_{i} \theta_{i, \mathcal{T}}} .
$$

Since $\theta_{i, \mathcal{T}} \geq \rho h_{\mathcal{T}}$, we finally obtain that

$$
\left|B_{i, m}^{(1)}(\boldsymbol{\Phi})\right|^{2} \leq C h_{\mathcal{T}} \underset{m \rightarrow+\infty}{\longrightarrow} 0, \quad \forall \boldsymbol{\Phi} \in C_{c}^{\infty}\left(Q_{T}\right) .
$$

Let us now consider $B_{i, m}^{(2)}(\boldsymbol{\Phi})$. To this end, remark first that the definition of $\boldsymbol{\Phi}_{\sigma}^{n}$ implies that

$$
\sum_{\sigma \in \mathcal{E}_{K}} m_{\sigma} \boldsymbol{\Phi}_{\sigma}^{n} \cdot \boldsymbol{n}_{K \sigma}=\int_{K} \boldsymbol{\nabla} \cdot \boldsymbol{\Phi}\left(t^{n}, \boldsymbol{x}\right) \mathrm{d} \boldsymbol{x}, \quad \forall K \in \mathcal{T}_{m}, \forall n \in\left\{1, \ldots, N_{m}\right\} .
$$

As a consequence, since $\mu_{i, \mathcal{T}_{m}, \Delta t_{m}}$ converges weakly towards $\mu_{i}$ and $c_{i, \mathcal{T}_{m}, \Delta t_{m}}$ converges strongly towards $c_{i}$ in $L^{2}\left(Q_{T}\right)$, we conclude that

$$
B_{i, m}^{(2)}(\boldsymbol{\Phi}) \underset{m \rightarrow+\infty}{\longrightarrow} \iint_{Q_{T}} \mu_{i} c_{i} \boldsymbol{\nabla} \cdot \boldsymbol{\Phi} \mathrm{d} \boldsymbol{x} \mathrm{d} t, \quad \forall \boldsymbol{\Phi} \in C_{c}^{\infty}\left(Q_{T}\right) .
$$


Thanks to (4.1), the term $B_{i, m}^{(3)}(\boldsymbol{\Phi})$ can be rewritten as

$$
B_{i, m}^{(3)}(\mathbf{\Phi})=\iint_{Q_{T}} \mu_{i, \mathcal{T}_{m}, \Delta t_{m}} \boldsymbol{\nabla}_{\mathcal{T}_{m}} c_{i, \mathcal{T}_{m}, \Delta t_{m}} \cdot \boldsymbol{\Phi}_{\mathcal{T}_{m}, \Delta t_{m}} \mathrm{~d} \boldsymbol{x} \mathrm{d} t
$$

The strong convergence of $\boldsymbol{\nabla}_{\mathcal{I}_{m}} c_{i, \mathcal{T}_{m}, \Delta t_{m}}$ towards $\boldsymbol{\nabla} c_{i}$ in $L^{2}\left(Q_{T}\right)^{2}$, the weak convergence of $\mu_{i, \mathcal{T}_{m}, \Delta t_{m}}$ towards $\mu_{i}$ and the uniform convergence of $\boldsymbol{\Phi}_{\mathcal{T}_{m}, \Delta t_{m}}$ towards $\boldsymbol{\Phi}$ yield

$$
B_{i, m}^{(3)}(\boldsymbol{\Phi}) \underset{m \rightarrow+\infty}{\longrightarrow} \iint_{Q_{T}} \mu_{i} \nabla c_{i} \cdot \boldsymbol{\Phi} \mathrm{d} \boldsymbol{x} \mathrm{d} t, \quad \forall \boldsymbol{\Phi} \in C_{c}^{\infty}\left(Q_{T}\right) .
$$

Introducing the quantities

$$
r_{i, K}^{n}=\frac{1}{m_{K}} \sum_{\sigma \in \mathcal{E}_{K}} m_{\sigma}\left(\bar{c}_{i, \sigma}^{n}-c_{i, K}^{n}\right)\left(\boldsymbol{\Phi}_{\sigma}^{n}-\boldsymbol{\Phi}_{K}^{n}\right) \cdot \boldsymbol{n}_{K \sigma}, \quad \forall K \in \mathcal{T}_{m}, \forall n \in\left\{1, \ldots, N_{m}\right\},
$$

and the corresponding functions $r_{i, \mathcal{T}_{m}, \Delta t_{m}}$ in $\mathcal{X}_{\mathcal{T}_{m}, \Delta t_{m}}$, the term $B_{i, m}^{(4)}(\boldsymbol{\Phi})$ rewrites

$$
B_{i, m}^{(4)}(\boldsymbol{\Phi})=\iint_{Q_{T}} \mu_{i, \mathcal{T}_{m}, \Delta t_{m}} r_{i, \mathcal{T}_{m}, \Delta t_{m}} \mathrm{~d} \boldsymbol{x} \mathrm{d} t
$$

Since $\mu_{i, \mathcal{T}_{m}, \Delta t_{m}}$ is uniformly bounded in $L^{2}\left(Q_{T}\right)$, proving that $r_{i, \mathcal{T}_{m}, \Delta t_{m}}$ tends to 0 in $L^{2}\left(Q_{T}\right)$ is enough to show that

$$
B_{i, m}^{(4)}(\boldsymbol{\Phi}) \underset{m \rightarrow+\infty}{\longrightarrow} 0, \quad \forall \boldsymbol{\Phi} \in C_{c}^{\infty}\left(Q_{T}\right) .
$$

Thanks to the regularity of the mesh $\mathcal{T}_{m}$, and more precisely to (2.13), there holds

$$
\left|r_{i, K}^{n}\right|^{2} \leq \frac{\ell^{\star}}{\left(m_{K}\right)^{2}} \sum_{\sigma \in \mathcal{E}_{K, \text { int }}}\left(m_{\sigma}\right)^{2}\left(\bar{c}_{i, \sigma}^{n}-c_{i, K}^{n}\right)^{2}\left|\Phi_{\sigma}^{n}-\boldsymbol{\Phi}_{K}^{n}\right|^{2} \leq \ell^{\star}\|D \boldsymbol{\Phi}\|_{\infty}^{2} \frac{\left(h_{K}\right)^{4}}{\left(m_{K}\right)^{2}} \sum_{\sigma \in \mathcal{E}_{K, \text { int }}} \tau_{K \sigma}\left(\bar{c}_{i, \sigma}^{n}-c_{i, K}^{n}\right)^{2} .
$$

Using estimate

$$
\sum_{n=1}^{N_{m}} \Delta t_{m} \sum_{K \in \mathcal{T}_{m}} \sum_{\sigma \in \mathcal{E}_{K, \text { int }}} \tau_{K \sigma}\left(\bar{c}_{i, \sigma}^{n}-c_{i, K}^{n}\right)^{2}=\sum_{n=1}^{N_{m}} \Delta t_{m} \sum_{\sigma \in \mathcal{E}_{\mathrm{int}, m}} \tau_{\sigma}\left(c_{i, K}^{n}-c_{i, L}^{n}\right)^{2} \leq T C_{1},
$$

one infers from (2.12) that

$$
\begin{aligned}
\left\|r_{i, \mathcal{T}_{m}, \Delta t_{m}}\right\|_{L^{2}\left(Q_{T}\right)}^{2} & =\sum_{n=1}^{N_{m}} \Delta t_{m} \sum_{K \in \mathcal{T}_{m}} m_{K}\left|r_{i, K}^{n}\right|^{2} \\
& \leq \ell^{\star}\|D \boldsymbol{\Phi}\|_{\infty}^{2} \sum_{n=1}^{N_{m}} \Delta t_{m} \sum_{K \in \mathcal{T}_{m}} \frac{\left(h_{K}\right)^{4}}{m_{K}} \sum_{\sigma \in \mathcal{E}_{K, \text { int }}} \tau_{K \sigma}\left(\bar{c}_{i, \sigma}^{n}-c_{i, K}^{n}\right)^{2} \leq T C_{1} \ell^{\star} \zeta\|D \boldsymbol{\Phi}\|_{\infty}^{2} h_{\mathcal{T}_{m}}^{2},
\end{aligned}
$$

so that $r_{i, \mathcal{T}_{m}, \Delta t_{m}}$ tends to 0 in $L^{2}\left(Q_{T}\right)$ and (4.9) holds.

Finally, we deduce from (4.5) to (4.9) that $\boldsymbol{V}_{i}=-c_{i} \boldsymbol{\nabla} \mu_{i}$ in the distributional sense, hence also in $L^{2}\left(Q_{T}\right)^{2}$.

We have now all the necessary material to conclude the proof of Theorem 2.4. This is the purpose of our last lemma. 


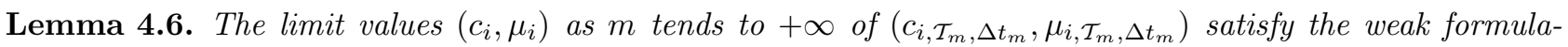
tions (1.16) for $i \in\{1,2\}$.

Proof. As a preliminary, let us first show that the functions $c_{i, \mathcal{D}_{m}, \Delta t_{m}}$ defined by

$$
c_{i, \mathcal{D}_{m}, \Delta t_{m}}(t, \boldsymbol{x})=\left\{\begin{array}{l}
c_{i, \sigma}^{n} \text { if }(t, \boldsymbol{x}) \in\left(t^{n-1}, t^{n}\right] \times D_{\sigma}, \sigma \in \mathcal{E}_{\mathrm{int}, m}, \\
c_{i, K}^{n} \text { if }(t, \boldsymbol{x}) \in\left(t^{n-1}, t^{n}\right] \times\left(K \backslash \bigcup_{\sigma \in \mathcal{E}_{K, \mathrm{int}}} D_{K, \sigma}\right),
\end{array}\right.
$$

converges strongly in $L^{2}\left(Q_{T}\right)$ towards $c_{i}$. Indeed, one has

$$
\begin{aligned}
\left\|c_{i, \mathcal{D}_{m}, \Delta t_{m}}-c_{i, \mathcal{T}_{m}, \Delta t_{m}}\right\|_{L^{2}\left(Q_{T}\right)}^{2} & =\sum_{n=1}^{N_{m}} \Delta t_{n} \sum_{K \in \mathcal{T}_{m}} \sum_{\sigma \in \mathcal{E}_{K, \text { int }}} m_{D_{K, \sigma}}\left(c_{i, K}^{n}-c_{i, \sigma}^{n}\right)^{2} \\
& \leq \sum_{n=1}^{N_{m}} \Delta t_{n} \sum_{\substack{\sigma \in \mathcal{E}_{\text {int, }, m} \\
\sigma=K \mid L}} m_{D_{\sigma}}\left(c_{i, K}^{n}-c_{i, L}^{n}\right)^{2} \leq \frac{T C_{1}}{2}\left(h_{\mathcal{T}_{m}}\right)^{2} \underset{m \rightarrow \infty}{\longrightarrow} 0 .
\end{aligned}
$$

Since $c_{i, \mathcal{T}_{m}, \Delta t_{m}}$ converges in $L^{2}\left(Q_{T}\right)$ towards $c_{i}$ as $m$ tends to $\infty$, then so does $c_{i, \mathcal{D}_{m}, \Delta t_{m}}$.

Let $\varphi \in C_{c}^{\infty}([0, T) \times \bar{\Omega})$, then denote by $\varphi_{K}^{n}=\varphi\left(t^{n}, \boldsymbol{x}_{K}\right)$ for all $K \in \mathcal{T}_{m}$ and all $n \in\left\{0, \ldots, N_{m}\right\}, m \geq 1$. Note that $\varphi_{K}^{N_{m}}=0$ for all $K \in \mathcal{T}_{m}$. Multiplying (2.3) by $\Delta t_{m} \varphi_{K}^{n-1}$ and summing over $K \in \mathcal{T}$ and $n \in\left\{1, \ldots, N_{m}\right\}$ leads to

$$
A_{i, m}+B_{i, m}+C_{i, m}+D_{i, m}=0
$$

where we have set

$$
\begin{aligned}
& A_{i, m}=\sum_{n=1}^{N_{m}} \sum_{K \in \mathcal{T}_{m}} m_{K} c_{i, K}^{n}\left(\varphi_{K}^{n-1}-\varphi_{K}^{n}\right)-\sum_{K \in \mathcal{T}_{m}} m_{K} c_{i, K}^{0} \varphi_{K}^{0}, \\
& B_{i, m}=\frac{1}{\eta_{i}} \sum_{n=1}^{N_{m}} \Delta t_{m} \sum_{\substack{\sigma \in \mathcal{E}_{\text {int }, m} \\
\sigma=K \mid L}} \tau_{\sigma} c_{i, \sigma}^{n}\left(\mu_{i, K}^{n}-\mu_{i, L}^{n}\right)\left(\varphi_{K}^{n-1}-\varphi_{L}^{n-1}\right), \\
& C_{i, m}=\frac{1}{\eta_{i}} \sum_{n=1}^{N_{m}} \Delta t_{m} \sum_{\substack{\sigma \in \mathcal{E}_{\text {int }, m} \\
\sigma=K \mid L}} \tau_{\sigma} c_{i, \sigma}^{n}\left(\Psi_{i, K}-\Psi_{i, L}\right)\left(\varphi_{K}^{n-1}-\varphi_{L}^{n-1}\right), \\
& D_{i, m}=\theta_{i, \mathcal{T}_{m}} \sum_{n=1}^{N_{m}} \Delta t_{m} \sum_{\substack{\sigma \in \mathcal{E}_{\text {int }, m} \\
\sigma=K \mid L}} \tau_{\sigma}\left(c_{i, K}^{n}-c_{i, L}^{n}\right)\left(\varphi_{K}^{n-1}-\varphi_{L}^{n-1}\right) .
\end{aligned}
$$

Classical arguments (see for instance [16]) allow to show that

$$
A_{i, m} \underset{m \rightarrow \infty}{\longrightarrow}-\iint_{Q_{T}} c_{i} \partial_{t} \varphi \mathrm{d} \boldsymbol{x} \mathrm{d} t-\int_{\Omega} c_{i}^{0} \varphi(0, \cdot) \mathrm{d} \boldsymbol{x},
$$

and, since $\theta_{i, \mathcal{T}_{m}}$ tends to $\theta_{i}$, that

$$
D_{i, m} \underset{m \rightarrow \infty}{\longrightarrow} \theta_{i} \iint_{Q_{T}} \nabla c_{i} \cdot \nabla \varphi \mathrm{d} \boldsymbol{x} \mathrm{d} t
$$


Using Taylor expansions, one shows that

$$
\left|\frac{\varphi_{K}^{n}-\varphi_{L}^{n}}{d_{\sigma}}+\frac{1}{m_{D_{\sigma}}} \int_{D_{\sigma}} \boldsymbol{\nabla} \varphi\left(t^{n}, \boldsymbol{x}\right) \cdot \boldsymbol{n}_{K L} \mathrm{~d} \boldsymbol{x}\right| \leq C d_{\sigma}, \quad \forall \sigma=K \mid L \in \mathcal{E}_{\mathrm{int}, m} .
$$

Therefore,

$$
B_{i, m}=-\frac{1}{\eta_{i}} \iint_{Q_{T}} \boldsymbol{V}_{i, \mathcal{D}_{m}, \Delta t_{m}} \cdot \boldsymbol{\nabla} \varphi \mathrm{d} \boldsymbol{x} \mathrm{d} t+B_{i, m}^{\prime}
$$

with

$$
\begin{aligned}
\left|B_{i, m}^{\prime}\right| \leq & \frac{C}{\eta_{i}} h_{\mathcal{T}_{m}} \sum_{n=1}^{N_{m}} \Delta t_{m} \sum_{\substack{\sigma \in \mathcal{E}_{\text {int }, m} \\
\sigma=K \mid L}} m_{\sigma} c_{i, \sigma}^{n}\left|\mu_{i, K}^{n}-\mu_{i, L}^{n}\right| \\
& +\frac{1}{\eta_{i}}\left|\sum_{n=1}^{N_{m}} \int_{t^{n-1}}^{t^{n}} \int_{\Omega}\left(\nabla \varphi(t, \boldsymbol{x})-\nabla \varphi\left(t^{n-1}, \boldsymbol{x}\right)\right) \cdot \boldsymbol{V}_{i, \mathcal{D}_{m}, \Delta t_{m}} \mathrm{~d} \boldsymbol{x} \mathrm{d} t\right| .
\end{aligned}
$$

Cauchy-Schwarz inequality together with Lemma 3.6, the regularity of $\varphi$ and the $L^{2}\left(Q_{T}\right)$ bound of $\boldsymbol{V}_{i, \mathcal{D}_{m}, \Delta t_{m}}$ show that $B_{i, m}^{\prime}$ tends to 0 as $m$ tends to $+\infty$, while Lemma 4.5 ensures that

$$
\lim _{m \rightarrow \infty} B_{i, m}=\iint_{Q_{T}} \frac{c_{i}}{\eta_{i}} \nabla \mu_{i} \cdot \nabla \varphi \mathrm{d} \boldsymbol{x} \mathrm{d} t
$$

Let us focus on the term $C_{i, m}$. Define the vectors

$$
\boldsymbol{W}_{i, \sigma}^{n}=2 \frac{\Psi_{i, K}-\Psi_{i, L}}{d_{\sigma}} \boldsymbol{n}_{K L}, \quad \forall \sigma=K \mid L \in \mathcal{E}_{\text {int }, m}, \forall n \in\left\{1, \ldots, N_{m}\right\},
$$

and the corresponding piecewise constant vector field

$$
\boldsymbol{W}_{i, \mathcal{D}_{m}, \Delta t_{m}}(t, \boldsymbol{x})=\boldsymbol{W}_{i, \sigma}^{n} \quad \text { if }(t, \boldsymbol{x}) \in\left(t^{n-1}, t^{n}\right] \times D_{\sigma}, \sigma \in \mathcal{E}_{\mathrm{int}, m},
$$

then it is shown in $[10,15]$ that $\boldsymbol{W}_{i, \mathcal{D}_{m}, \Delta t_{m}}$ converges weakly in $L^{2}\left(Q_{T}\right)$ towards $-\nabla \Psi_{i}$. Therefore, $c_{i, \mathcal{D}_{m}, \Delta t_{m}} \boldsymbol{W}_{i, \mathcal{D}_{m}, \Delta t_{m}}$ converges weakly in $L^{p}\left(Q_{T}\right)$ towards $-c_{i} \nabla \Psi_{i}$ for all $p<2$. Proceeding as for $B_{i, m}$, one shows that

$$
\lim _{m \rightarrow \infty} C_{i, m}=\iint_{Q_{T}} \frac{c_{i}}{\eta_{i}} \nabla \Psi_{i} \cdot \nabla \varphi \mathrm{d} \boldsymbol{x} \mathrm{d} t .
$$

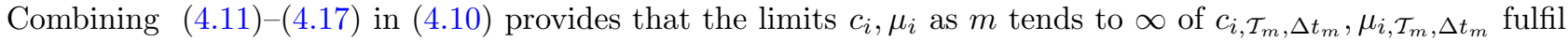
the weak formulation (1.16).

Remark 4.7. A natural way to discretize (1.2) would have been to use a Scharfetter-Gummel scheme [30] in (2.3). This scheme degenerates into the upstream mobility scheme proposed in [9] in the deep quench limit $\theta_{i, \mathcal{T}}=0$. Almost all our analysis can be adapted to this scheme excepted Lemma 4.5. More precisely, we are not able to prove that the term $B_{i, m}^{(1)}(\boldsymbol{\Phi})$ appearing in the proof of Lemma 4.5 tends to 0 as $m$ tends to $+\infty$, which possibly breaks the consistency of the scheme.

\section{NumericAl RESUltS}

In this section, we present different simulations to illustrate the behavior of the finite-volume scheme presented in Section 2.2. To solve this nonlinear system we use a Newton-Raphson based iterative method. More precisely, the unknowns $\left(c_{2, K}^{n}\right)_{K \in \mathcal{T}}$ are eliminated thanks to the relation (2.6), so that the nonlinear system to be solved 


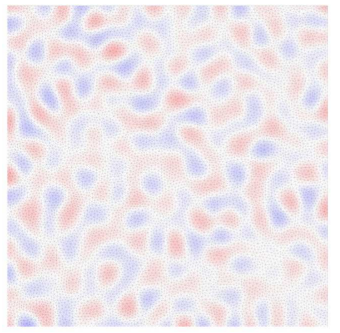

(A)

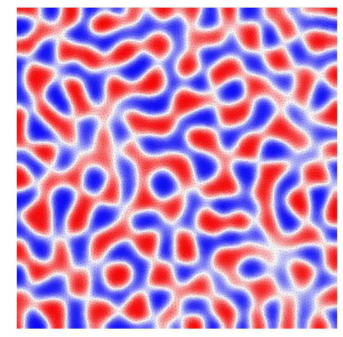

(B)

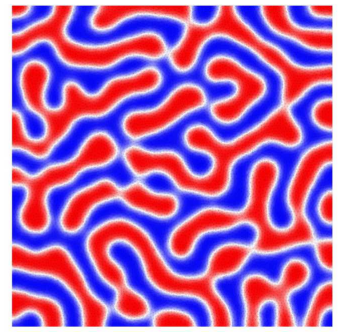

(c)

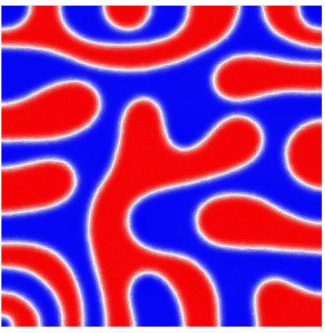

(D)

Figure 2. Spinodal decomposition without external potential, $c_{1}^{0}(\boldsymbol{x})=0.5+r(\boldsymbol{x})$. [(A) $t=$ 0.005. (B) $t=0.01$. (C) $t=0.02$. (D) $t=0.2$.

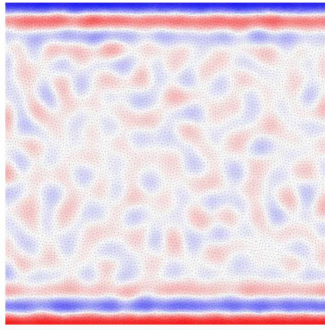

(A)

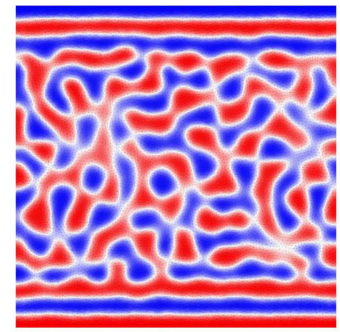

(B)

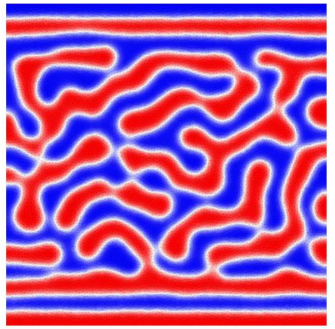

(c)

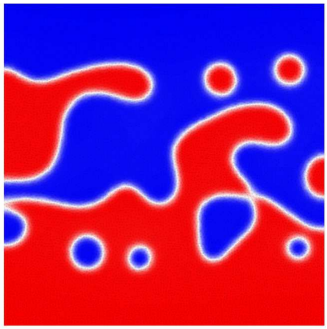

(D)

Figure 3. Spinodal decomposition with external potentials, $c_{1}^{0}(\boldsymbol{x})=0.5+r(\boldsymbol{x})$. [(A) $t=0.005$. (B) $t=0.01$. (C) $t=0.02$. (D) $t=0.2$.]

at each time step involves 3 unknowns $c_{1, K}^{n}, \mu_{1, K}^{n}$ and $\mu_{2, K}^{n}$ per cell $K \in \mathcal{T}$. The iterative method stops as soon as the $\ell_{2}$ norm of the Newton increment is smaller than $10^{-6}$. The updated concentration variables are projected on the set $[\epsilon, 1-\epsilon]^{\mathcal{T}}$, with $\epsilon=10^{-10}$, which is reasonable in view of Lemma 3.2.

In each case the domain $\Omega$ is the square $(0,1)^{2}$. The mesh is made of 23330 conforming triangles. The mesh size is approximately equal to 0.017 and the time step is fixed to $\Delta t=10^{-4}$. We choose as parameters $\alpha=0.0002$, $\kappa=1.45, \theta_{1}=\theta_{2}=0.35, \rho=1$ and $\nu_{1}=\nu_{2}=1$. We plot the concentration $c_{1}$ and we can observe in blue the concentration $c_{1}=0$, in red $c_{1}=1$ and in white $c_{1}=0.5$.

First we consider the spinodal decomposition test case. The initial saturation $c_{1}^{0}$ is a random initial concentration with a fluctuation, that is $c_{1}^{0}(\boldsymbol{x})=0.5+r(\boldsymbol{x})$ where $r \ll 1$ is a small random perturbation. We compare the case without any external potential, that is $\Psi_{1}=\Psi_{2}=0$, in Figure 2 with the case where the external potential are given by $\Psi_{i}(\boldsymbol{x})=-\rho_{i} \boldsymbol{g} \cdot \boldsymbol{x}$ where the gravity is $\boldsymbol{g}=-0.98 \boldsymbol{e}_{y}$ and the densities $\rho_{1}=5$ and $\rho_{2}=1$ in Figure 3. Note that in both cases we have exactly the same initial data. We want to observe the influence of the gravity on the phase separation dynamics.

At the very beginning (see Figs. $2 \mathrm{a}$ and $3 \mathrm{a}$ ), as the state $c_{1}=0.5$ is slightly disturbed, the two pure phases $c_{1}=0$ and $c_{1}=1$ quickly spontaneously separate. However in the second case, as the phase $c_{1}$ is heavier, we can clearly observe in Figure $3 \mathrm{a}$ the influence of the external potentials at the bottom and the top. Then the pure phases gradually come together to form larger patterns (see Figs. 2b-2d and Figs. 3b-3d). Furthermore, it can be seen that even if the external potentials have a very strong influence on the phase separation dynamics at the top and the bottom, in a short time, the phase separation dynamic is very similar at the center of the domain (see Figs. 2a-2c and Figs. 3a-3c). But, in a longer time, the influence of external potentials on the entire phase separation dynamics can be observed in Figures 2d and 3d. 


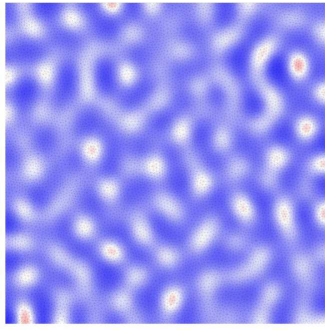

(A)

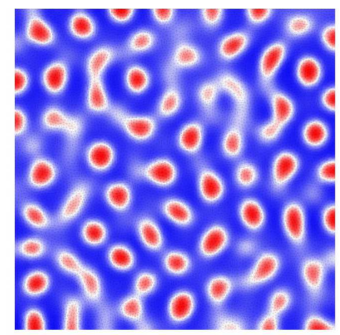

(B)

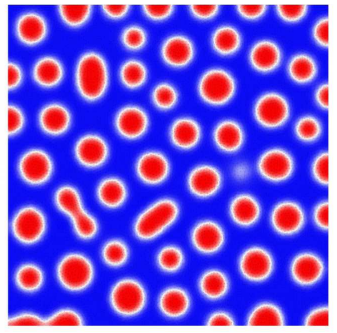

(c)

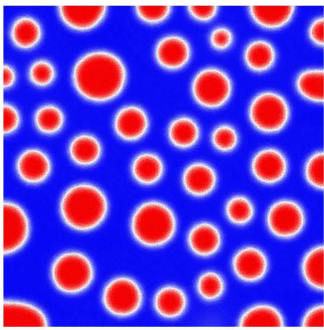

(D)

Figure 4. Spinodal decomposition without external potential, $c_{1}^{0}(\boldsymbol{x})=0.3+r(\boldsymbol{x})$. (A) $t=$ 0.011. (B) $t=0.015$. (C) $t=0.05$. (D) $t=0.2$.

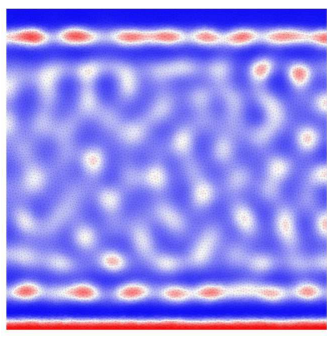

(A)

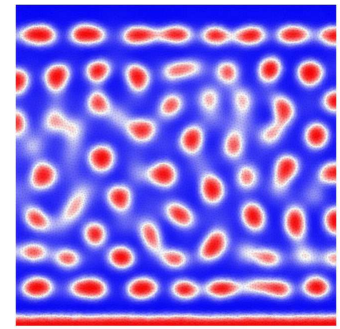

(B)

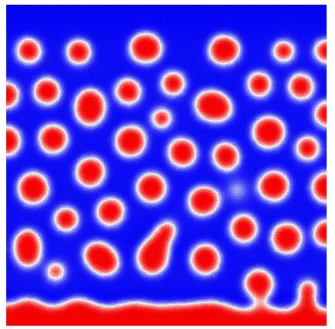

(C)

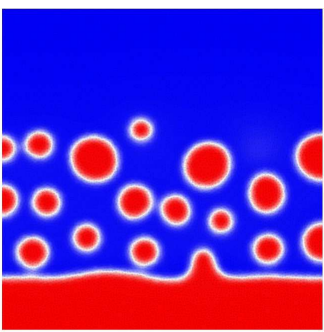

(D)

Figure 5. Spinodal decomposition with external potentials, $c_{1}^{0}(\boldsymbol{x})=0.3+r(\boldsymbol{x})$. (A) $t=0.011$. (B) $t=0.015$. (C) $t=0.05$. (D) $t=0.2$.

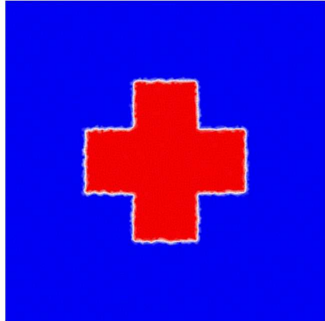

(A)

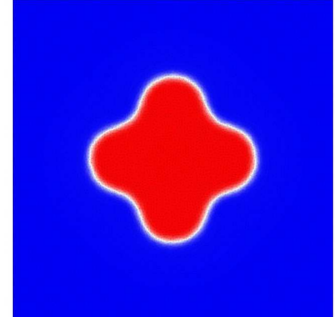

(B)

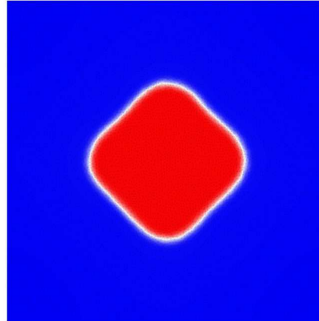

(c)

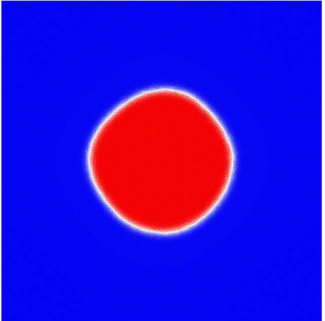

(D)

Figure 6. Cross as initial data without external potential. (A) $t=0$. (B) $t=0.02$. (C) $t=0.07$. (D) $t=0.2$.

Now we keep exactly the same data but we change the initial condition by favouring the pure phase $c_{1}=0$ and choosing $c_{1}^{0}(\boldsymbol{x})=0.3+r(\boldsymbol{x})$.

First of all we can see in Figures 4a and 5a that, as expected, the pure phase $c_{1}=1$, and thus, the phase separation dynamics appears later than in Figures 2a and 3a. Moreover, since the phase $c_{1}=0$ is preponderant, a collection of circular droplets of the pure phase $c_{1}=1$ can be observed over a long period of time (see Figs. 4c, $4 \mathrm{~d}$ and $5 \mathrm{c}, 5 \mathrm{~d}$ ). Our observations on Figures 2 and 3 concerning the influence of the external potentials are still valid with this choice of initial profiles.

We consider now a second test case. The initial concentration is a cross in the middle of the domain presented in Figure 6a. 


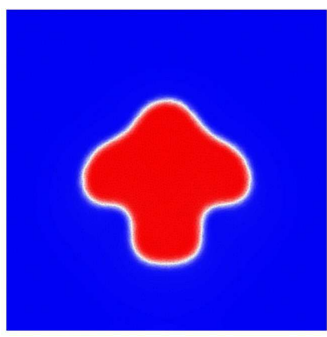

(A)

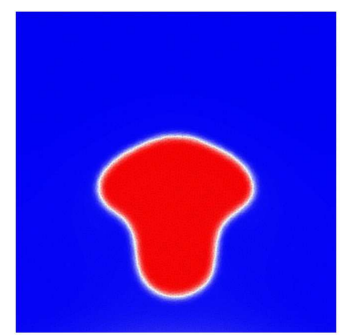

(B)

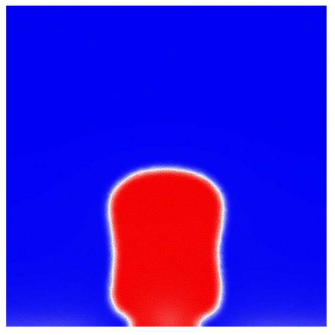

(C)

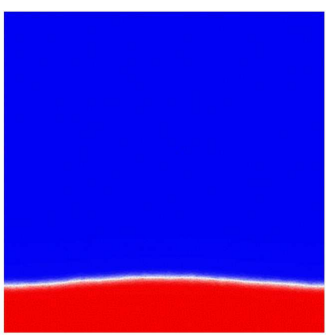

(D)

Figure 7. Cross as initial data with external potentials. (A) $t=0.02$. (B) $t=0.07$. (C) $t=0.15$. (D) $t=0.5$.

Here again we start with the case without external potentials. We know that in this case the Cahn-Hilliard model preserves the volume while minimizing the perimeter and thus as seen in Figure 6 the cross evolves into a circle.

Now, we want to observe the influence of the gravity when we add the external potentials $\Psi_{i}(\boldsymbol{x})=-\rho_{i} \boldsymbol{g} \cdot \boldsymbol{x}$. Since $\rho_{1}=5>1=\rho_{2}$, as one might expect, we observe in Figure 7 that the cross, that is the pure phase $c_{1}=1$, is drawn down. Thus, although the volume is still preserved, the final state is no longer a circle but a strip at the bottom of the domain.

\section{Appendix A. TeChnicAl Lemma}

For $\lambda \in[0,1]$, let $f_{\lambda}$ and $p_{\lambda}$ be defined as in (3.29), and let $H_{\lambda}: \mathbb{R} \rightarrow[0,+\infty]$ be the convex function defined by

$$
H_{\lambda}(c)=\int_{1}^{c} p_{\lambda}(a) \mathrm{d} a= \begin{cases}c \log \frac{1-\lambda}{1+\lambda}+\lambda & \text { if } c \leq \frac{1-\lambda}{2} \\ c \log \frac{2 c}{1+\lambda}-c+\frac{1+\lambda}{2} & \text { if } c \in\left[\frac{1-\lambda}{2}, \frac{1+\lambda}{2}\right], \\ 0 & \text { if } c \geq \frac{1+\lambda}{2}\end{cases}
$$

if $\lambda<1$ and

$$
H_{1}(c)= \begin{cases}+\infty & \text { if } c<0 \\ c \log c-c+1 & \text { if } c \in[0,1] \\ 0 & \text { if } c \geq 1\end{cases}
$$

One readily checks that

$$
\lim _{\lambda \succ 1} H_{\lambda}(c)=H_{1}(c), \quad \forall c \in \mathbb{R} .
$$

Let us establish the following lemma, which is used in the proof of Proposition 3.9.

Lemma A.1. For all $\beta>0$, there exists $C_{\beta}$ depending only on $\beta$ such that

$$
\frac{1-\lambda}{2}\left(c-\frac{1}{2}\right)^{2}+H_{\lambda}(c)+H_{\lambda}(1-c) \geq \beta\left|c-\frac{1}{2}\right|-C_{\beta}, \quad \forall \lambda \in[0,1], \forall c \in \mathbb{R} .
$$

Proof. Assume that there exists a nonnegative super-linear function $\Upsilon: \mathbb{R}_{+} \rightarrow \mathbb{R}_{+}$such that

$$
\frac{1-\lambda}{2}\left(c-\frac{1}{2}\right)^{2}+H_{\lambda}(c)+H_{\lambda}(1-c) \geq \Upsilon\left(\left|c-\frac{1}{2}\right|\right), \quad \forall \lambda \in[0,1], \forall c \in \mathbb{R},
$$

and

$$
\lim _{u \rightarrow+\infty} \frac{\Upsilon(u)}{u}=+\infty .
$$


Then, we proceed as in the proof of Lemma 3.3 from [6] to establish (A.3). More precisely, we infer from (A.5) that for all $\beta>0$, there exists $r_{\beta}>0$ such that

$$
u \geq r_{\beta} \Longrightarrow \Upsilon(u) \geq \beta u .
$$

Since $\Upsilon(u)$ is assumed to be nonnegative, one has

$$
\Upsilon(u) \geq \beta u-\beta r_{\beta}, \quad \forall u \geq 0,
$$

so that (A.4) implies (A.3). Therefore, the problem reduces to show that such an $\Upsilon$ exists.

As a preliminary, we remark that the left-hand side of (A.4) is invariant by replacing $c$ by $(1-c)$, so that if we establish (A.4) for $c \geq \frac{1}{2}$, it will also hold true for $c \leq \frac{1}{2}$. Define

$$
\Upsilon(u)=\inf _{\lambda \in[0,1)}\left\{\frac{1-\lambda}{2}|u|^{2}+H_{\lambda}\left(u+\frac{1}{2}\right)+H_{\lambda}\left(\frac{1}{2}-u\right)\right\} \geq 0, \quad \forall u \geq 0,
$$

then (A.4) automatically holds. It only remains to check that so does (A.5). The above definition of $\Upsilon$ can be reformulated as

$$
\Upsilon(u)=\inf _{\lambda \in[0,1)} \mathcal{Z}_{u}(\lambda), \quad \forall u \geq 0,
$$

where, recalling the expression (A.1) of $H_{\lambda}, \mathcal{Z}_{u}$ is the $C^{1}$ function defined on $[0,1)$ by

$$
\mathcal{Z}_{u}(\lambda)= \begin{cases}\frac{1-\lambda}{2} u^{2}+\left(\frac{1}{2}-u\right) \log \frac{1-\lambda}{1+\lambda}+\lambda & \text { if } \lambda \leq 2 u, \\ \frac{1-\lambda}{2} u^{2}+\lambda-\log (1+\lambda)+\left(\frac{1}{2}+u\right) \log (1+2 u)+\left(\frac{1}{2}-u\right) \log (1-2 u) & \text { if } \lambda \geq 2 u,\end{cases}
$$

for all $u \geq 0$ and $\lambda \in[0,1)$. An elementary study of this function shows that $\mathcal{Z}_{u}$ reaches its minimum on $[0,1]$ at

$$
\lambda^{\star}(u)= \begin{cases}0 & \text { if } u \leq 4, \\ \sqrt{1-\frac{4 u-2}{u^{2}-2}} & \text { if } u \geq 4 .\end{cases}
$$

Using this expression in the above expression of $\mathcal{Z}_{u}(\lambda)$, we can explicit $\Upsilon(u)$ as

$$
\Upsilon(u)= \begin{cases}\frac{u^{2}}{2} & \text { if } u \leq 4 \\ \Upsilon_{1}(u)+\Upsilon_{2}(u)+\lambda^{\star}(u) & \text { if } u \geq 4\end{cases}
$$

where we have set

$$
\Upsilon_{1}(u)=\frac{u^{2}}{2}\left(1-\lambda^{\star}(u)\right), \quad \text { and } \quad \Upsilon_{2}(u)=\left(u-\frac{1}{2}\right) \log \frac{1+\lambda^{\star}(u)}{1-\lambda^{\star}(u)} .
$$

Noticing that $\lambda^{\star}(u) \sim 1-\frac{2}{u}$ as $u$ tends to $+\infty$, one obtains that $\Upsilon_{1}(u) \sim u$ behaves linearly at infinity. However, $\Upsilon(u)$ is super-linear, i.e. (A.5) holds since $\Upsilon_{2}(u) \sim u \log u$ as $u \rightarrow+\infty$.

\section{Appendix B. A Discrete Aubin-Simon lemma}

The goal of this appendix is to state a ready to use discrete Aubin-Simon lemma adapted to our TPFA finite volume framework. Several time-compactness results for the numerical approximations have been proposed in the last decade, see for instance $[2,3,11,18,19]$. The statement we propose here is tailored for our application rather than aiming at being general. It requires the introduction of a sequence of regular discretizations of $Q_{T}$ as introduced before Theorem 2.4, the corresponding spaces $\mathcal{X}_{\mathcal{T}_{m}, \Delta t_{m}}$ and the approximate gradient operators $\nabla_{\mathcal{T}_{m}}$ as in Proposition 4.2 . 
Lemma B.1. Let $\left(\mathcal{T}_{m}, \mathcal{E}_{m},\left(\boldsymbol{x}_{K}\right)_{K \in \mathcal{T}_{m}}, \Delta t_{m}\right)_{m>1}$ be a sequence of regular discretizations of $Q_{T}$, and let $\left(u_{\mathcal{T}_{m}, \Delta t_{m}}\right)_{m \geq 1}$ be a sequence of piecewise constant functions with $u_{\mathcal{T}_{m}, \Delta t_{m}} \in \mathcal{X}_{\mathcal{T}_{m}, \Delta t_{m}}$ for all $m \geq 1$ such that:

(i) the sequences $\left(u_{\mathcal{T}_{m}, \Delta t_{m}}\right)_{m>1}$ and $\left(\nabla_{\mathcal{T}_{m}} u_{\mathcal{T}_{m}, \Delta t_{m}}\right)_{m>1}$ are bounded in $L^{2}\left(Q_{T}\right)$ and $L^{2}\left(Q_{T}\right)^{2}$ respectively;

(ii) For all $\varphi_{\mathcal{T}_{m}, \Delta t_{m}} \in \mathcal{X}_{\mathcal{T}_{m}}$, there holds

$$
\sum_{n=1}^{N_{m}} \sum_{K \in \mathcal{T}_{m}} m_{K}\left(u_{K}^{n}-u_{K}^{n-1}\right) \varphi_{K}^{n} \leq C\left\|\nabla_{\mathcal{T}_{m}} \varphi_{\mathcal{T}_{m}, \Delta t_{m}}\right\|_{L^{2}\left(Q_{T}\right)^{2}},
$$

for some $C$ not depending on $m$.

Then, up to a subsequence, $u_{\mathcal{T}_{m}, \Delta t_{m}}$ converges strongly in $L_{\mathrm{loc}}^{2}\left(Q_{T}\right)$ towards $u \in L^{2}\left((0, T) ; H^{1}(\Omega)\right)$ as $m$ tends to $+\infty$.

Proof. Let us first notice that, owing to Proposition 4.2, the properties (i) above ensures that $u_{\mathcal{T}_{m}, \Delta t_{m}}$ converges weakly in $L^{2}\left(Q_{T}\right)$ towards some $u \in L^{2}\left((0, T) ; H^{1}(\Omega)\right)$. Now, let us check that the assumptions of Proposition 3.8 from [3] are fulfilled. Assumption $\left(\mathbf{A}_{t}\right)$ of [3] is automatically fulfilled for one-step time discretizations (including the backwards Euler scheme). Besides, the condition $\left(\mathbf{A}_{\mathbf{x}} 1\right)$ of [3], which is about the compactness w.r.t. space of sequences with bounded gradients holds, as established in [17] for SUSHI finite volume approximations. Since the $L^{2}\left(Q_{T}\right)^{2}$ norm was used in the right-hand side of (B.1) (in opposition to the $L^{\infty}\left(Q_{T}\right)^{2}$ norm suggested by [3]), it is sufficient to establish the relaxed version of condition $\left(\mathbf{A}_{\mathbf{x}} 3\right)$ of [3]: for any $\varphi \in C_{c}^{\infty}(\Omega)$, define

$$
\varphi_{K}=\frac{1}{m_{K}} \int_{K} \varphi \mathrm{d} \boldsymbol{x}, \quad \forall K \in \mathcal{T}_{m}, \forall m \geq 1,
$$

and $\varphi_{\mathcal{T}_{m}}(\boldsymbol{x})=\varphi_{K}$ if $\boldsymbol{x} \in K$, then there exists some $C>0$ such that

$$
\left\|\nabla_{\mathcal{T}_{m}} \varphi_{\mathcal{T}_{m}}\right\|_{L^{2}\left(Q_{T}\right)^{2}} \leq C\|\nabla \varphi\|_{L^{2}\left(Q_{T}\right)^{2}}, \quad \forall m \geq 1 .
$$

This constant $C$ is exactly the constant $C_{2}$ introduced before, the inequality being established in Lemma 9.4 of [16].

We are in position to make use of Proposition 3.8 from [3], which shows that for all $\varphi \in C_{c}^{\infty}\left(Q_{T}\right)$,

$$
\iint_{Q_{T}}\left|u_{\mathcal{T}_{m}, \Delta t_{m}}\right|^{2} \varphi \mathrm{d} \boldsymbol{x} \mathrm{d} t \underset{m \rightarrow \infty}{\longrightarrow} \iint_{Q_{T}}|u|^{2} \varphi \mathrm{d} \boldsymbol{x} \mathrm{d} t .
$$

This allows in particular to show that

$$
\iint_{Q_{T}}\left|u_{\mathcal{T}_{m}, \Delta t_{m}}-u\right|^{2} \varphi \mathrm{d} \boldsymbol{x} \mathrm{d} t \underset{m \rightarrow \infty}{\longrightarrow} 0,
$$

hence the claimed $L_{\mathrm{loc}}^{2}\left(Q_{T}\right)$ convergence property.

Lemma B.1 can be directly used on $\left(c_{i, \mathcal{T}_{m}, \Delta t_{m}}\right)_{m>1}$. Indeed, while $c_{i, \mathcal{T}_{m}, \Delta t_{m}}$ is uniformly bounded in $L^{\infty}\left(Q_{T}\right)$ thus in $L^{2}\left(Q_{T}\right)$ too, $\nabla_{\mathcal{T}_{m}} c_{i, \mathcal{T}_{m}, \Delta t_{m}}$ is uniformly bounded in $L^{\infty}\left((0, T) ; L^{2}(\Omega)\right)^{2}$, thus in $L^{2}\left(Q_{T}\right)^{2}$ too. Moreover, using (2.3), Cauchy-Schwarz inequality and $0 \leq c_{i, \sigma}^{n} \leq 1$, one gets that

$$
\begin{aligned}
\sum_{n=1}^{N_{m}} \sum_{K \in \mathcal{T}_{m}} m_{K}\left(c_{i, K}^{n}-c_{i, K}^{n-1}\right) \varphi_{K}^{n} & \leq \frac{1}{\sqrt{\eta_{i}}}\left(\sum_{n=1}^{N_{m}} \Delta t_{m} \mathfrak{D}_{\mathcal{T}_{m}}\left(\boldsymbol{c}^{n}, \boldsymbol{\mu}^{n}\right)\right)^{1 / 2}\left\|\boldsymbol{\nabla}_{\mathcal{T}_{m}} \varphi_{\mathcal{T}_{m}, \Delta t_{m}}\right\|_{L^{2}\left(Q_{T}\right)^{2}} \\
& \leq C\left\|\nabla_{\mathcal{T}_{m}} \varphi_{\mathcal{T}_{m}, \Delta t_{m}}\right\|_{L^{2}\left(Q_{T}\right)^{2}} .
\end{aligned}
$$

Acknowledgements. The authors acknowledge the support of the French National Research Agency (ANR) through grant ANR-13-JS01-0007-01 (project GEOPOR). C. Cancès also acknowledges support from Labex CEMPI (ANR-11-LABX0007-01). The authors also thank the anonymous referees for their help in improving the presentation. 


\section{REFERENCES}

[1] L. Ambrosio, N. Gigli and G. Savaré, Gradient flows in metric spaces and in the space of probability measures, 2nd edition. Lectures in Mathematics ETH Zürich. Birkhäuser Verlag, Basel (2008).

[2] B. Andreianov, Time compactness tools for discretized evolution equations and applications to degenerate parabolic PDEs, edited by J. Fořt, J. Fürst, J. Halama, R. Herbin and F. Hubert. In: Finite Volumes for Complex Applications. VI. Problems \& Perspectives. Springer Proceedings in Mathematics. Springer, Berlin, Heidelberg (2011) 21-29.

[3] B. Andreianov, C. Cancès and A. Moussa, A nonlinear time compactness result and applications to discretization of degenerate parabolic-elliptic PDEs. J. Funct. Anal. 273 (2017) 3633-3670.

[4] J.-D. Benamou, Y. Brenier and K. Guittet, Numerical analysis of a multi-phasic mass transport problem. In: Recent Advances in the Theory and Applications of Mass Transport. Vol. 353 of Contemporary Mathematics Amer. Math. Soc., Providence, RI (2004) 1-17.

[5] M. Bessemoulin-Chatard, C. Chainais-Hillairet and F. Filbet, On discrete functional inequalities for some finite volume schemes. IMA J. Numer. Anal. 35 (2015) 1125-1149.

[6] C. Cancès and C. Guichard, Numerical analysis of a robust free energy diminishing finite volume scheme for parabolic equations with gradient structure. Found. Comput. Math. 17 (2017) 1525-1584.

[7] C. Cancès and D. Matthes, Construction of a two-phase flow with singular energy by gradient flow methods. HAL: hal-02510535 (2020).

[8] C. Cancès, D. Matthes and F. Nabet, A two-phase two-fluxes degenerate Cahn-Hilliard model as constrained Wasserstein gradient flow. Arch. Ration. Mech. Anal. 233 (2019) 837-866.

[9] C. Cancès and F. Nabet, Finite volume approximation of a degenerate immiscible two-phase flow model of Cahn-Hilliard type, In: Finite Volumes for Complex Applications VIII - Methods and Theoretical Aspects: edited by C. Cancès and P. Omnes. FVCA 8, Lille, France, June 2017. number 199 in Proceedings in Mathematics and Statistics. Springer International Publishing, Cham (2017) 431-438.

[10] C. Chainais-Hillairet, J.-G. Liu and Y.-J. Peng, Finite volume scheme for multi-dimensional drift-diffusion equations and convergence analysis. ESAIM: M2AN 37 (2003) 319-338.

[11] X. Chen, A. Jüngel and J.-G. Liu, A note on Aubin-Lions-Dubinskii lemmas. Acta Appl. Math. 133 (2013) $33-43$.

[12] K. Deimling, Nonlinear Functional Analysis. Springer-Verlag, Berlin (1985).

[13] J. Droniou, R. Eymard, T. Gallouët, C. Guichard and R. Herbin, The Gradient Discretisation Method. In: Vol. 42 of Mathématiques et Applications. Springer International Publishing (2018).

[14] C.M. Elliott and H. Garcke, On the Cahn-Hilliard equation with degenerate mobility. SIAM J. Math. Anal. 27 (1996) $404-423$.

[15] R. Eymard and T. Gallouët, H-convergence and numerical schemes for elliptic problems. SIAM J. Numer. Anal. 41 (2003) $539-562$.

[16] R. Eymard, T. Gallouët and R. Herbin, Finite volume methods. In: Handbook of numerical analysis. edited by P.G. Ciarlet, et al. North-Holland, Amsterdam (2000) 713-1020.

[17] R. Eymard, T. Gallouët and R. Herbin, Discretization of heterogeneous and anisotropic diffusion problems on general nonconforming meshes sushi: a scheme using stabilization and hybrid interfaces. IMA J. Numer. Anal. 30 (2010) $1009-1043$.

[18] T. Gallouët, Discrete functional analysis tools for some evolution equations. Comput. Methods Appl. Math. 18 (2018) $477-493$.

[19] T. Gallouët and J.-C. Latché, Compactness of discrete approximate solutions to parabolic PDEs - application to a turbulence model Comm. Pure Appl. Anal. 11 (2012) 2371-2391.

[20] P. Gladbach, E. Kopfer and J. Maas, Scaling limits of discrete optimal transport. SIAM J. Math. Anal. 52 (2020) $2759-2802$.

[21] A. Glitzky and J.A. Griepentrog, Discrete Sobolev-Poincaré inequalities for Voronoi finite volume approximations. SIAM J. Numer. Anal. 48 (2010) 372-391.

[22] G. Grün and M. Rumpf, Nonnegativity preserving convergent schemes for the thin film equation. Numer. Math. 87 (2000) 113-152.

[23] R. Jordan, D. Kinderlehrer and F. Otto, The variational formulation of the Fokker-Planck equation. SIAM J. Math. Anal. 29 (1998) 1-17.

[24] J. Leray and J. Schauder, Topologie et équations fonctionnelles. Ann. Sci. École Norm. Sup. 51 (1934) 45-78.

[25] S. Lisini, D. Matthes and G. Savaré, Cahn-Hilliard and thin film equations with nonlinear mobility as gradient flows in weighted-Wasserstein metrics. J. Differ. Equ. 253 (2012) 814-850.

[26] J. Maas, Gradient flows of the entropy for finite Markov chains. J. Funct. Anal. 261 (2011) 2250-2292.

[27] J. Maas and D. Matthes, Long-time behavior of a finite volume discretization for a fourth order diffusion equation. Nonlinearity 29 (2016) 1992-2023.

[28] A. Mielke, A gradient structure for reaction-diffusion systems and for energy-drift-diffusion systems. Nonlinearity 24 (2011) 1329-1346.

[29] F. Otto and E. Weinan, Thermodynamically driven incompressible fluid mixtures. J. Chem. Phys. 107 (1997) $10177-10184$.

[30] D.L. Scharfetter and H.K. Gummel, Large-signal analysis of a silicon read diode oscillator. IEEE Trans. Electron Dev. 16 (1969) 64-77.

[31] C. Villani, Optimal transport. In: Vol. 338 of Grundlehren der Mathematischen Wissenschaften [Fundamental Principles of Mathematical Sciences]. Springer-Verlag, Berlin (2009). Old and new.

[32] W. E and P. Palffy-Muhoray, Phase separation in incompressible systems. Phys. Rev. E 55 (1997) R3844-R3846. 